\title{
Dispersion variance for transport in heterogeneous porous media
}

\author{
Marco Dentz ${ }^{1}$ and Felipe P. J. de Barros ${ }^{2}$ \\ Received 4 October 2012; revised 8 April 2013; accepted 22 April 2013; published 17 June 2013.
}

[1] We study dispersion in heterogeneous porous media for solutes evolving from point-like and extended source distributions in $d=2$ and $d=3$ spatial dimensions. The impact of heterogeneity on the dispersion behavior is captured by a stochastic modeling approach that represents the spatially fluctuating flow velocity as a spatial random field. We focus here on the sample-to-sample fluctuations of the dispersion coefficients about their ensemble mean. For finite source sizes, the definition of dispersion coefficients in single realizations is not unique. We consider dispersion measures that describe the extension of the solute distribution, as well as dispersion coefficients that quantify the solute spreading relative to injection points of the partial plumes that constitute the solute distribution. While the ensemble averages of these dispersion quantities may be identical, their fluctuation behavior is found to be different. Using a perturbation approach in the fluctuations of the random flow field, we derive explicit expressions for the temporal evolution of the variances of the dispersion coefficients between realizations. Their evolution is governed by the typical dispersion time over the characteristic heterogeneity scale and the dimensions of the source distribution. We find that the dispersion variance decreases toward zero with time in $d=3$ spatial dimensions, while in $d=2$ it converges toward a finite long time value that is independent of the source dimensions.

Citation: Dentz, M., and F. P. J. de Barros (2013), Dispersion variance for transport in heterogeneous porous media, Water Resour. Res., 49, 3443-3461, doi:10.1002/wrcr.20288.

\section{Introduction}

[2] Heterogeneity in the hydraulic medium properties impacts on the way dissolved substances are transported. Spatial fluctuations in hydraulic conductivity lead to solute spreading that is much larger than expected from localscale dispersion [Gelhar et al., 1992]. Breakthrough curves may show early or late solute arrivals, and spatial concentration profiles may exhibit forward and backward tails that cannot be explained based on advective-dispersive transport models for equivalent homogeneous media [Boggs et al., 1992; Haggerty et al., 2000; Berkowitz et al., 2006]. In short, heterogeneity impacts on the transport dynamics. On the other hand, the incomplete knowledge of the heterogeneity details on scales smaller than the relevant observation scales renders the transport behavior uncertain [Rubin, 2003; Tartakovsky and Winter, 2008].

[3] Stochastic modeling is a powerful tool to quantify the heterogeneity-induced average transport dynamics, as well as the uncertainty about this behavior. This approach models the spatially heterogeneous hydraulic conductivity

\footnotetext{
${ }^{1}$ Institute of Environmental Assessment and Water Research (IDAEA), Spanish National Research Council (CSIC), Barcelona, Spain.

${ }^{2}$ Sonny Astani Department of Civil and Environmental Engineering, University of Southern California, Los Angeles, California, USA

Corresponding author: M. Dentz, Institute of Environmental Assessment and Water Research (IDAEA), Spanish National Research Council (CSIC), 08034 Barcelona, Spain. (marco.dentz@csic.es)

field as a random field; this means that a concrete medium is considered a realization of an ensemble of media. The stochasticity of the medium renders the flow and transport behavior stochastic as well. The large-scale transport behavior is obtained by averaging of the behavior of the quantity of interest in single media over all possible medium realizations. This approach allows one to quantify the full transport statistics, in principle, and specifically the mean behavior and its variance [Graham and McLaughlin, 1989; Neuman, 1993; Cushman et al., 1994; Fiori and Dagan, 2000; Fiori, 2001]. The quantities of interest can be the concentration distribution, solute breakthrough curves, as well as the center of mass velocity and the growth rate of the spatial plume extension.

[4] The quantification of the evolution of the effective transport behavior in terms of the mean concentration and breakthrough curves has been studied intensively over the last two decades [e.g., Berkowitz et al., 2006; Neuman and Tartakovsky, 2008; Dentz et al., 2010]. In this paper, however, we focus on the center of mass velocity and apparent dispersion coefficients, their ensemble averages, and the uncertainty about them.

[5] The increase in solute spreading due to spatial heterogeneity has been quantified in terms of ensemble and effective dispersion coefficients. Ensemble dispersion coefficients are defined in terms of the spatial variance of the average solute concentration [Gelhar and Axness, 1983; Dagan, 1984; Neuman et al., 1987; Dagan, 1988; Rubin et al., 1999], while the effective dispersion coefficients are defined in terms of the average over the spatial variances in individual medium realizations [Kitanidis, 1988; Dagan, 1990, 
1991; Rajaram and Gelhar, 1993; Bellin et al., 1996; Attinger et al., 1999; Dentz et al., 2000a, 2000b]. As a consequence, the ensemble dispersion coefficients quantify an artificial spreading effect that is due to the sample-to-sample fluctuation of the center of mass position of the solute plume. Thus, in general, ensemble dispersion overestimates the plume spreading behavior in single realizations. Under certain conditions, ensemble and effective coefficients converge to the asymptotic macrodispersion coefficient in the limit of asymptotically large times, or infinite source size [Rajaram and Gelhar, 1993; Dentz et al., 2000b]. Both quantities are defined as ensemble averages, and therefore, they capture the mean spreading behavior.

[6] A key question is how well these coefficients characterize the spreading in a single aquifer realization. We focus on the average of the dispersion behavior in single aquifer realizations, which yields, in average, effective dispersion coefficients. The dispersion behavior in single medium realizations is measured in terms of suitably defined apparent dispersion coefficients, which in a stochastic framework, represent realizations of a stochastic process. The fluctuation of these observables about their mean value is a measure for the heterogeneity-induced uncertainty. If their variance tends toward zero in the limit of large times, they are called self-averaging [Bouchaud and Georges, 1990]. The notion of self-averaging needs to be distinguished from ergodicity. In short, a process is ergodic if the ensemble average and a suitably defined space or time average coincide, while an observable is self-averaging if its sample-to-sample fluctuations, measured by its variance, tend to zero with time [Bouchaud and Georges, 1990]. Therefore, self-averaging implies that the full disorder configuration has been sampled by the plume at large times. Note that the underlying stochastic process, here hydraulic conductivity, may be ergodic, while dispersion and the center of mass velocity may not be self-averaging. This is the case, for example, for transport in stratified formations [Clincy and Kinzelbach, 2001].

[7] Dagan [1990] considers the sample-to-sample fluctuations of the first and second centered spatial moments of a solute plume, for purely advective transport in a stratified medium. He focuses on the behavior of the variance of the second centered moment in function of the initial plume size. Fiori and Janković [2005] investigate this behavior for $d=2$ and $d=3$ dimensional heterogeneous media using numerical simulations. Clincy and Kinzelbach [2001] study the sample-to-sample fluctuations of apparent dispersion coefficients for stratified velocity fields in the presence of diffusion. They investigate the temporal behavior of the variance of the apparent dispersion coefficients and find that dispersion is not self-averaging for this system. Eberhard [2004] studies the self-averaging properties for a solute transported in $d=3$ dimensional quasi-periodic and Gaussian random velocity fields. This study finds that dispersion in Gaussian velocity fields is self-averaging and dispersion in quasi-periodic fields is not. Later, Suciu et al. [2006], Eberhard et al. [2007], and Suciu et al. [2008, 2009] investigated the self-averaging behavior of dispersion in such random velocity fields using numerical simulations. de Barros and Rubin [2011] quantified the dispersion variance as a function of the plume scale and the homogenization scale and used a statistical inference method to estimate the full probability density function (PDF) of the dispersion coefficients. In fact, the PDF of dispersion may be used to estimate the PDF of concentration point values as proposed in the mapping approach by Dentz and Tartakovsky [2010]. In the context of solute mixing, Cirpka et al. [2011a, 2011b] provide first-order estimates on the uncertainty of the transverse effective dispersion coefficient for the fringe of a steady-state contaminant plume.

[8] In this paper, we study the self-averaging properties of dispersion in a heterogeneous porous medium. This work is motivated by the question how well dispersion coefficients that are defined as ensemble averages describe solute spreading in a single medium realization. This addresses a fundamental question of the stochastic approach. Furthermore, this analysis provides a measure for uncertainty in estimated plume extensions which is important for risk assessment studies and for the interpretation of dispersion data from field and laboratory experiments. A related issue that we will discuss in this work is definition and meaning of dispersion measures in single realizations and the related fluctuation behavior.

[9] In fact, studying the self-averaging properties of dispersion requires a sound definition of apparent dispersion coefficients in single medium realizations. We define absolute, effective, and relative dispersion coefficients within single medium realizations.

[10] The absolute dispersion coefficients are derived from second centered moments of the concentration distribution. Absolute dispersion has been routinely considered in the literature as a dispersion measure [Kitanidis, 1988; Dagan, 1990]. It quantifies the growth rate of the extension of the solute distribution in single realizations. The definitions of the effective and relative dispersion coefficients rely on the fact that the concentration distribution $c(\mathbf{x}, t)$ can be expressed in terms of a superposition of a continuum of partial plumes $g\left(\mathbf{x}, t \mid \mathbf{x}^{\prime}\right)$ that originate from instantaneous point injections at $\mathbf{x}=\mathbf{x}^{\prime}$,

$$
c(\mathbf{x}, t)=\int d \mathbf{x}^{\prime} \rho\left(\mathbf{x}^{\prime}\right) g\left(\mathbf{x}, t \mid \mathbf{x}^{\prime}\right),
$$

where $\rho(\mathbf{x})=c(\mathbf{x}, t=0)$. The effective and relative dispersion coefficients are defined in terms of the moments of the partial plumes $g\left(\mathbf{x}, t \mid \mathbf{x}^{\prime}\right)$ [e.g., Fiori, 2001; Dentz and Carrera, 2007; Zavala-Sanchez et al., 2009] relative to the injection point $\mathbf{x}^{\prime}$ and thus measure the average dispersion behavior in the plume rather than the plume extension.

[11] The objective of this work is to analyze the selfaveraging behavior of these dispersion quantities in terms of their respective ensemble variances. These ensemble variances quantify how well dispersion measures describe the actual dispersion in a single disorder realization and provide uncertainty estimates. The dispersion variance provides also an indicator for the mixing state of the transport system. As the system is better mixed the variance decreases because the differences between realizations are expected to decrease. Specifically, we study the role of the dimensionality of space and the extension of the initial plume on the self-averaging properties of these dispersion measures. We employ a perturbation theory approach to derive explicit analytical expressions for the dispersion 
variances, which implies that the presented results are strictly valid only for moderate heterogeneity.

[12] This paper is organized as follows. The next section presents the flow and transport model and the stochastic modeling approach. Section 3 defines the absolute, effective, and relative dispersion coefficient and discusses their physical meaning, the relations between them, and their respective ensemble averages. Section 4 develops the perturbation theory used in section 5 to obtain explicit analytical expressions for the variances of the center of mass velocity and the respective dispersion coefficients. Section 6 discusses the temporal evolution of the dispersion variances in $d=2$ and $d=3$ spatial dimensions and their dependence on the dimensions of the initial plumes.

\section{Basics}

[13] This section introduces the flow and transport model the following study is based on, as well as the stochastic modeling approach to quantify the impact of spatial heterogeneity in the hydraulic medium properties.

\subsection{Flow and Transport Model}

[14] The evolution of the concentration $c(\mathbf{x}, t)$ of a dissolved substance in a heterogeneous porous medium can be described by the advection-dispersion equation [Bear, 1972]

$$
\frac{\partial c(\mathbf{x}, t)}{\partial t}+\nabla \cdot[\mathbf{u}(\mathbf{x}) c(\mathbf{x}, t)]-\nabla \cdot[\mathbf{D} \nabla c(\mathbf{x}, t)]=0
$$

where $\mathbf{u}(\mathbf{x})$ is the flow velocity, and $\mathbf{D}$ represents the localscale dispersion tensor, which is assumed to be constant and diagonal, $D_{i j}=D_{i} \delta_{i j}$. Porosity is assumed to be constant and set to one. We consider an infinite $d$-dimensional transport domain with natural boundary conditions for $c(\mathbf{x}, t)$, and the normalized initial condition is $c(\mathbf{x}, t=0)=\rho(\mathbf{x})$. For technical convenience, in this work, we employ a Gaussian initial distribution [Attinger et al., 1999; Dentz et al., 2000b] given by

$$
\rho(\mathbf{x})=\prod_{i=1}^{d} \frac{\exp \left(-\left(x_{i}^{2} / 2 L_{i}^{2}\right)\right)}{\sqrt{2 \pi L_{i}^{2}}},
$$

where $L_{i}$ is the source extension in $i$ direction. The Green's function $g\left(\mathbf{x}, t \mid \mathbf{x}^{\prime}\right)$ satisfies (2) for the initial condition

$$
g\left(\mathbf{x}, t=0 \mid \mathbf{x}^{\prime}\right)=\delta\left(\mathbf{x}-\mathbf{x}^{\prime}\right) .
$$

[15] The concentration distribution $c(\mathbf{x}, t)$ is given in terms of the Green's function $g\left(\mathbf{x}, t \mid \mathbf{x}^{\prime}\right)$ by equation (1). As outlined in section 1 , the total plume consists of a superposition of partial plumes that originate at $\mathbf{x}=\mathbf{x}^{\prime}$ and whose distributions are given by the Green's functions $g\left(\mathbf{x}, t \mid \mathbf{x}^{\prime}\right)$.

[16] The transport problem can be formulated equivalently in a Lagrangian framework that describes the motion of solute particles in terms of the Langevin equation

$$
\frac{\mathrm{d} \mathbf{x}\left(t \mid \mathbf{x}^{\prime}\right)}{\mathrm{d} t}=\mathbf{u}\left[\mathbf{x}\left(t \mid \mathbf{x}^{\prime}\right)\right]+\sqrt{2 \mathbf{D}} \cdot \zeta(t),
$$

where $\zeta(t)$ denotes a Gaussian white noise characterized by zero mean and unit variance. The particle trajectories $\mathbf{x}\left(t \mid \mathbf{x}^{\prime}\right)$ originate from $\mathbf{x}\left(t=0 \mid \mathbf{x}^{\prime}\right)=\mathbf{x}^{\prime}$ where the initial points $\mathbf{x}^{\prime}$ are distributed according to $\rho\left(\mathbf{x}^{\prime}\right)$. The Green's function $g\left(\mathbf{x}, t \mid \mathbf{x}^{\prime}\right)$ reads in terms of the particle trajectories $\mathbf{x}\left(t \mid \mathbf{x}^{\prime}\right)$ as

$$
g\left(\mathbf{x}, t \mid \mathbf{x}^{\prime}\right)=\left\langle\delta\left[\mathbf{x}-\mathbf{x}\left(t \mid \mathbf{x}^{\prime}\right)\right]\right\rangle,
$$

where the angular brackets denote the noise average over all realizations of $\xi(t)$. In the following, we will use both the Eulerian and Lagrangian formulations when it is required for illustration.

[17] The flow velocity is divergence free, $\nabla \cdot \mathbf{u}(\mathbf{x})=0$ and follows the Darcy equation [Bear, 1972]

$$
\mathbf{u}(\mathbf{x})=-K(\mathbf{x}) \nabla h(\mathbf{x})
$$

where $K(\mathbf{x})$ denotes the (isotropic) hydraulic conductivity, and $h(\mathbf{x})$ denotes the hydraulic head. Taking the divergence of (7) gives the flow equation

$$
\nabla^{2} h(\mathbf{x})+\nabla f(\mathbf{x}) \cdot \nabla h(\mathbf{x})=0,
$$

where $f(\mathbf{x})=\ln [K(\mathbf{x})]$ is log-conductivity.

\subsection{Stochastic Model}

[18] Spatial heterogeneity in $K(\mathbf{x})$ is quantified using a stochastic modeling approach in which $f(\mathbf{x})$ is represented as a multi-Gaussian spatial random field. We assume that $f(\mathbf{x})$ is statistically stationary so that it can be fully characterized by its constant mean $\overline{f(\mathbf{x})}$ and the correlation function of its fluctuations $f^{\prime}(\mathbf{x})=f(\mathbf{x})-\overline{f(\mathbf{x})}$, which is given by $C\left(\mathbf{x}-\mathbf{x}^{\prime}\right)=\overline{f^{\prime}(\mathbf{x}) f^{\prime}\left(\mathbf{x}^{\prime}\right)}$. The overbar denotes the ensemble average over all realizations of $f(\mathbf{x})$. The variance of $f(\mathbf{x})$ is given by $\sigma^{2}=C(0)$. In the following, we employ a Gaussian covariance function for the fluctuations of $f(\mathbf{x})$ such that

$$
C\left(\mathbf{x}-\mathbf{x}^{\prime}\right)=\sigma^{2} \prod_{i=1}^{d} \exp \left[-\frac{\left(x_{i}-x_{i}^{\prime}\right)^{2}}{2 l_{i}^{2}}\right]
$$

where $l_{i}$ denotes the correlation length in $i$ direction. We apply a constant hydraulic gradient $\mathbf{G}=\nabla \overline{h(\mathbf{x})}$ that is aligned with the 1 direction of the coordinate system, $\mathbf{G}=G \mathbf{e}_{1}$. By the linear approximation, $\mathbf{u}(\mathbf{x})$ follows the same statistics as $f^{\prime}(\mathbf{x})$ and therefore is approximately a stationary Gaussian random field [e.g., Gelhar and Axness, 1983]. Its mean value is given by $\overline{\mathbf{u}(\mathbf{x})}=\overline{\mathbf{u}}=K_{g} G \mathbf{e}_{1}$ where $K_{g}=\exp (\bar{f})$ is the geometric mean of $K$. The covariance function of the fluctuations, $\mathbf{u}^{\prime}(\mathbf{x})=\mathbf{u}(\mathbf{x})-\overline{\mathbf{u}}$, in Fourier space is defined by

$$
\tilde{C}_{i j}(\mathbf{k})=\bar{u}^{2} p_{i}(\mathbf{k}) p_{j}(\mathbf{k}) \tilde{C}(\mathbf{k})
$$

with $\mathbf{k}$ denoting the wave number vector, $p_{i}(\mathbf{k})=$ $\delta_{1 i}-k_{1} k_{i} / k^{2}$, and $\tilde{C}(\mathbf{k})$ representing the log-conductivity covariance function in Fourier space [Gelhar and Axness, 1983; Rubin, 2003]. The integral transform-inverse pair is defined as follows: 


$$
\begin{aligned}
& \tilde{f}(\mathbf{k})=\int \mathrm{d} \mathbf{x} \exp (i \mathbf{k} \cdot \mathbf{x}) f(x) \\
& f(\mathbf{x})=\int_{k} \exp (-i \mathbf{k} \cdot \mathbf{x}) \tilde{f}(\mathbf{k}) .
\end{aligned}
$$

[19] Here and in the following, we employ the shorthand notation

$$
\int \frac{\mathrm{d} \mathbf{k}}{(2 \pi)^{d}} \cdots \equiv \int_{k} \cdots
$$

[20] The correlation length $l_{i}$, the dispersion coefficients $D_{i i}$, and the mean flow velocity $\bar{u}$ define the following time scales and dimensionless numbers: the advection time scale $\tau_{u}=l_{1} / \bar{u}$ measures the typical time for advective solute transport over one correlation length. The dispersion time scales $\tau_{D_{i}}=l_{i}^{2} / D_{i i}$ measure the time for dispersive transport over the same distance. The Péclet numbers $P e_{i}=\tau_{D_{i}} / \tau_{u}=\bar{u} l_{i}^{2} /\left(l_{1} D_{i i}\right)$ compare the strength of advective and dispersive transport mechanisms.

\section{Dispersion Concepts}

[21] We focus here on the variance of the center of mass velocity and the dispersion coefficients between realizations of hydraulic conductivity. In the following, we illustrate different dispersion concepts for single medium realizations (see also Table 1). We define three effective dispersion measures: "absolute effective," "effective for point injection," and "effective relative." For the upcoming sections, we will denote these measures as "absolute," "effective," and "relative" correspondingly. We discuss the relations between the different dispersion coefficients and determine their respective ensemble averages. Finally, we define the observables of interest in this paper, that is, the variances of the respective dispersion coefficients between realizations of the random flow field $\mathbf{u}(\mathbf{x})$.

\subsection{Absolute Dispersion}

[22] The absolute moments of the (normalized) concentration distribution $c(\mathbf{x}, t)$ are defined as

$$
\begin{gathered}
m_{i}^{a}(t)=\int \mathrm{d} \mathbf{x} x_{i} c(\mathbf{x}, t)=\int \mathrm{d} \mathbf{x}^{\prime} \rho\left(\mathbf{x}^{\prime}\right)\left\langle x_{i}\left(t \mid \mathbf{x}^{\prime}\right)\right\rangle \\
m_{i j}^{a}(t)=\int \mathrm{d} \mathbf{x} x_{i} x_{j} c(\mathbf{x}, t)=\int \mathrm{d} \mathbf{x}^{\prime} \rho\left(\mathbf{x}^{\prime}\right)\left\langle x_{i}\left(t \mid \mathbf{x}^{\prime}\right) x_{j}\left(t \mid \mathbf{x}^{\prime}\right)\right\rangle .
\end{gathered}
$$

[23] The equality of the expressions on the respective right-hand sides results from (1) and (6). The particle trajectories are given by integration of (5) as

$$
x_{i}\left(t \mid \mathbf{x}^{\prime}\right)=x_{i}^{\prime}+\hat{x}_{i}\left(t \mid \mathbf{x}^{\prime}\right),
$$

where we defined the trajectories relative to the injection point at $\mathbf{x}^{\prime}$,

$$
\hat{x}_{i}\left(t \mid \mathbf{x}^{\prime}\right)=\int_{0}^{t} \mathrm{~d} t^{\prime}\left\{\mathbf{u}\left[\mathbf{x}\left(t^{\prime} \mid \mathbf{x}^{\prime}\right)\right]+\sqrt{2 D_{i}} \xi_{i}\left(t^{\prime}\right)\right\} .
$$

[24] The absolute second centered moments, which are a measure for the extension of the solute distribution, are defined by

$$
\kappa_{i j}^{a}(t)=m_{i j}^{a}(t)-m_{i}^{a}(t) m_{j}^{a}(t)
$$

[25] The absolute center of mass velocity $v_{i}^{a}(t)$ measures the temporal rate of change of the first moment $m_{i}^{a}(t)$,

$$
v_{i}^{a}(t)=\frac{\mathrm{d} m_{i}^{a}(t)}{\mathrm{d} t} .
$$

[26] The absolute dispersion coefficients $D_{i j}^{a}(t)$ are defined as half the temporal rate of change of the absolute second centered moments $\kappa_{i j}^{a}(t)$ as

$$
D_{i j}^{a}(t)=\frac{1}{2} \frac{\mathrm{d} \kappa_{i j}^{a}(t)}{\mathrm{d} t}
$$

[27] These observables can be expressed in terms of the

\begin{tabular}{|c|c|c|c|c|}
\hline Single Realization & Equation & Ensemble Average & Equation & Concept \\
\hline$D_{i j}^{a}(t)$ & (20) & $\bar{D}_{i j}^{r}(t)$ & (46) & Absolute dispersion \\
\hline$D_{i j}^{e}\left(t \mid \mathbf{x}^{\prime}\right)$ & (28) & $\bar{D}_{i j}^{e}(t)$ & (47) & Local effective dispersion \\
\hline$D_{i j}^{e}(t)$ & (30) & $\bar{D}_{i j}^{e}(t)$ & (47) & Global effective dispersion \\
\hline$D_{i j}^{r}(t)$ & (37) & $\bar{D}_{i j}^{r}(t)$ & (46) & Relative dispersion \\
\hline$D_{i j}$ & (2) & N/A & & Local dispersion \\
\hline
\end{tabular}
generating function

$$
\chi^{a}(\mathbf{k}, t)=\int \mathrm{d} \mathbf{x e x p}(i \mathbf{k} \cdot \mathbf{x}) c(\mathbf{x}, t)=\tilde{c}(\mathbf{k}, t),
$$

see definition (11) of the Fourier transform. They read as

$$
\begin{gathered}
v_{i}^{a}(t)=-i \frac{\mathrm{d}}{\mathrm{d} t} \frac{\partial}{\partial k_{i}} \ln \left[\chi^{a}(\mathbf{k}, t)\right]_{\mathbf{k}=0} \\
D_{i j}^{a}(t)=-\frac{1}{2} \frac{\mathrm{d}}{\mathrm{d} t} \frac{\partial^{2}}{\partial k_{i} \partial k_{j}} \ln \left[\chi^{a}(\mathbf{k}, t)\right]_{\mathbf{k}=0},
\end{gathered}
$$

[28] This can be checked by inspection.

Table 1. Dispersion Concepts 


\subsection{Effective Dispersion}

[29] In order to define an effective dispersion coefficient, we consider now the moments of the Green's function $g\left(\mathbf{x}, t \mid \mathbf{x}^{\prime}\right)$ relative to the initial position $\mathbf{x}^{\prime}$

$$
\begin{gathered}
\hat{m}_{i}\left(t \mid \mathbf{x}^{\prime}\right)=\int \mathrm{d} \mathbf{x}\left(x_{i}-x_{i}^{\prime}\right) g\left(\mathbf{x}, t \mid \mathbf{x}^{\prime}\right)=\left\langle\hat{x}_{i}\left(t \mid \mathbf{x}^{\prime}\right)\right\rangle \\
\hat{m}_{i j}\left(t \mid \mathbf{x}^{\prime}\right)=\int \mathrm{d} \mathbf{x}\left(x_{i}-x_{i}^{\prime}\right)\left(x_{j}-x_{j}{ }^{\prime}\right) g\left(\mathbf{x}, t \mid \mathbf{x}^{\prime}\right)=\left\langle\hat{x}_{i}\left(t \mid \mathbf{x}^{\prime}\right) \hat{x}_{j}\left(t \mid \mathbf{x}^{\prime}\right)\right\rangle .
\end{gathered}
$$

[30] where the second equalities in the respective equations follow from (1), (6), and (17). Similar definitions for the spatial moments can be found in Fiori [2001] where the spatial moments for a point source were used to quantify concentration statistics and reduce the corresponding uncertainty in predictions. The local second centered moments $\hat{\kappa}_{i j}\left(t \mid \mathbf{x}^{\prime}\right)$ of a plume that evolves from a point injection at $\mathbf{x}^{\prime}$ are given by

$$
\hat{\kappa}_{i j}\left(t \mid \mathbf{x}^{\prime}\right)=\hat{m}_{i j}\left(t \mid \mathbf{x}^{\prime}\right)-\hat{m}_{i}\left(t \mid \mathbf{x}^{\prime}\right) \hat{m}_{j}\left(t \mid \mathbf{x}^{\prime}\right) .
$$

[31] The center of mass velocity for a partial plume originating in $\mathbf{x}^{\prime}$ is defined by

$$
\hat{v}_{i}\left(t \mid \mathbf{x}^{\prime}\right)=\frac{\mathrm{d} \hat{m}_{i}\left(t \mid \mathbf{x}^{\prime}\right)}{\mathrm{d} t}=\frac{\mathrm{d}\left\langle\hat{x}_{i}\left(t \mid \mathbf{x}^{\prime}\right)\right\rangle}{\mathrm{d} t}
$$

and the local effective dispersion coefficient for a partial plume is

$$
D_{i j}^{e}\left(t \mid \mathbf{x}^{\prime}\right)=\frac{1}{2} \frac{\mathrm{d} \hat{\kappa}_{i j}\left(t \mid \mathbf{x}^{\prime}\right)}{\mathrm{d} t} .
$$

[32] Global effective second centered moments are defined by

$$
\kappa_{i j}^{e}(t)=\int \mathrm{d} \mathbf{x}^{\prime} \rho\left(\mathbf{x}^{\prime}\right) \hat{\kappa}_{i j}\left(t \mid \mathbf{x}^{\prime}\right)
$$

[33] And the global effective dispersion coefficient for a plume evolving from the initial distribution $\tilde{\rho}(\mathbf{k})$ is given by

$$
D_{i j}^{e}(t)=\frac{1}{2} \frac{\mathrm{d} \kappa_{i j}^{e}(t)}{\mathrm{d} t}=\int \mathrm{d} \mathbf{x}^{\prime} \rho\left(\mathbf{x}^{\prime}\right) D_{i j}^{e}\left(t \mid \mathbf{x}^{\prime}\right) .
$$

[34] The above quantity measures the effective spreading of the solute with respect to the relative center of mass of each partial plume. It can also be seen as a spatial average of the local effective dispersion coefficients over the initial distribution.

[35] The global effective dispersion coefficients can be written in terms of the generating function

$$
\hat{\chi}\left(\mathbf{k}, t \mid \mathbf{x}^{\prime}\right)=\exp \left(-i \mathbf{k} \cdot \mathbf{x}^{\prime}\right) \tilde{g}\left(\mathbf{k}, t \mid \mathbf{x}^{\prime}\right)
$$

as

$$
D_{i j}^{e}(t)=-\frac{1}{2} \frac{\mathrm{d}}{\mathrm{d} t} \int \mathrm{d} \mathbf{x}^{\prime} \rho\left(\mathbf{x}^{\prime}\right) \frac{\partial^{2}}{\partial k_{i} \partial k_{j}} \ln \left[\hat{\chi}\left(\mathbf{k}, t \mid \mathbf{x}^{\prime}\right)\right]_{\mathbf{k}=0}
$$

[36] This can be checked by inspection.

\subsection{Relative Dispersion}

[37] The relative first and second moments of the concentration distribution $c(\mathbf{x}, t)$ are defined as

$$
\begin{aligned}
& m_{i}^{r}(t)=\int \mathrm{d} \mathbf{x}^{\prime} \rho\left(\mathbf{x}^{\prime}\right) \hat{m}_{i}\left(t \mid \mathbf{x}^{\prime}\right) \\
& m_{i j}^{r}(t)=\int \mathrm{d} \mathbf{x}^{\prime} \rho\left(\mathbf{x}^{\prime}\right) \hat{m}_{i j}\left(t \mid \mathbf{x}^{\prime}\right),
\end{aligned}
$$

and consequently, the corresponding relative second centered moment is given by

$$
\kappa_{i j}^{r}(t)=m_{i j}^{r}(t)-m_{i}^{r}(t) m_{j}^{r}(t) .
$$

[38] The relative center of mass velocity is defined by

$$
v_{i}^{r}(t)=\frac{\mathrm{d} m_{i}^{r}(t)}{\mathrm{d} t}
$$

and the relative dispersion coefficients are defined as

$$
D_{i j}^{r}(t)=\frac{1}{2} \frac{\mathrm{d}}{\mathrm{d} t} \kappa_{i j}^{r}(t)
$$

[39] It measures solute spreading with respect to the relative center of mass of the concentration distribution. As in the previous cases, the relative dispersion coefficients can be expressed in terms of the generating function

$$
\chi^{r}(\mathbf{k}, t)=\int \mathrm{d} \mathbf{x}^{\prime} \rho\left(\mathbf{x}^{\prime}\right) \hat{\chi}\left(\mathbf{k}, t \mid \mathbf{x}^{\prime}\right)
$$

[40] They are given by

$$
D_{i j}^{r}(t)=-\frac{1}{2} \frac{\mathrm{d}}{\mathrm{d} t} \frac{\partial^{2}}{\partial k_{i} \partial k_{j}} \ln \left[\chi^{r}(\mathbf{k}, t)\right]_{\mathbf{k}=\mathbf{0}} .
$$

\subsection{Relations Between the Observables}

[41] The difference between $\kappa_{i j}^{r}(t)$ and $\kappa_{i j}^{e}(t)$ is given by

$$
\begin{aligned}
\kappa_{i j}^{r}(t)-\kappa_{i j}^{e}(t)= & \int \mathrm{d} \mathbf{x}^{\prime} \int \mathrm{d} \mathbf{x}^{\prime \prime} \rho\left(\mathbf{x}^{\prime}\right)\left[\hat{m}_{i}\left(t \mid \mathbf{x}^{\prime}\right)-\rho\left(\mathbf{x}^{\prime \prime}\right) \hat{m}_{i}\left(t \mid \mathbf{x}^{\prime \prime}\right)\right] \\
& \times\left[\hat{m}_{j}\left(t \mid \mathbf{x}^{\prime}\right)-\rho\left(\mathbf{x}^{\prime \prime}\right) \hat{m}_{j}\left(t \mid \mathbf{x}^{\prime \prime}\right)\right]
\end{aligned}
$$

[42] It measures the variance of the center of mass positions between the partial plumes that constitute the concentration distribution $c(\mathbf{x}, t)$ (see equation (1)).

[43] Let us consider now the differences between the absolute and relative moments. The difference between the absolute and relative first moments (14) and (33) is given by

$$
m_{i}^{a}(t)-m_{i}^{r}(t)=\int \mathrm{d} \mathbf{x}^{\prime} \rho\left(\mathbf{x}^{\prime}\right) x_{i}^{\prime} \equiv m_{i}^{a}(0)
$$

which is the constant center of mass of the initial plume. Thus, the absolute and relative center of mass velocities are 
identical, $v_{i}(t)=v_{i}^{a}(t)=v_{i}^{r}(t)$. The difference between the absolute and relative second centered moments (18) and (29) thus reads as

$$
\begin{aligned}
\kappa_{i j}^{a}(t)-\kappa_{i j}^{r}(t)= & \kappa_{i j}^{a}(0)+\int \mathrm{d} \mathbf{x}^{\prime} \rho\left(\mathbf{x}^{\prime}\right)\left\{\left[x_{i}^{\prime}-m_{i}^{a}(0)\right] \hat{m}_{j}\left(t \mid \mathbf{x}^{\prime}\right)\right. \\
& \left.+\left[x_{j}^{\prime}-m_{j}^{a}(0)\right] \hat{m}_{i}\left(t \mid \mathbf{x}^{\prime}\right)\right\} .
\end{aligned}
$$

[44] The first term is the spatial variance of the initial plume $\rho(\mathbf{x})$, and the second term represents cross terms between the initial particle positions and the relative particle trajectories. The second term was also identified by Sposito and Dagan [1994] and studied numerically by Fiori and Janković [2005] and Suciu et al. [2008, 2009]. Notice that the papers by Suciu et al. take into account local dispersion. Based on (42), we obtain for the difference between the absolute and relative dispersion coefficients (20) and (30)

$$
\begin{aligned}
D_{i j}^{a}(t)-D_{i j}^{r}(t)= & \frac{1}{2} \int \mathrm{d} \mathbf{x}^{\prime} \rho\left(\mathbf{x}^{\prime}\right)\left\{\left[x_{i}{ }^{\prime}-m_{i}^{a}(0)\right]\left\langle\hat{v}_{j}\left(t \mid \mathbf{x}^{\prime}\right)\right\rangle\right. \\
& \left.+\left[x_{j}{ }^{\prime}-m_{j}^{a}(0)\right]\left\langle\hat{v}_{i}\left(t \mid \mathbf{x}^{\prime}\right)\right\rangle\right\},
\end{aligned}
$$

where we used (24) and (27). By definition, the initial values of the effective and relative dispersion coefficients are equal to the local dispersion coefficients, $D_{i j}^{e}(0)=$ $D_{i j}^{r}(0)=D_{i j}$. The initial value of the absolute dispersion coefficients, however, is given by the finite value

$$
\begin{aligned}
D_{i j}^{a}(0)= & D_{i j}+\frac{1}{2} \int \mathrm{d} \mathbf{x}^{\prime} \rho\left(\mathbf{x}^{\prime}\right)\left\{\left[x_{i}^{\prime}-m_{i}^{a}(0)\right] u_{j}\left(\mathbf{x}^{\prime}\right)\right. \\
& \left.+\left[x_{j}^{\prime}-m_{j}^{a}(0)\right] u_{i}\left(\mathbf{x}^{\prime}\right)\right\} .
\end{aligned}
$$

[45] This behavior is counterintuitive as one would expect that a meaningful measure for dispersion reduces to the local-scale dispersion coefficients at time $t=0$.

[46] Notice that all dispersion quantities are identical in case of a point source, for which $\rho(\mathbf{x})=\delta\left(\mathbf{x}-\mathbf{x}^{\prime}\right)$. This can be seen by inserting the latter into (40) and (42).

\subsection{Ensemble Averages}

[47] The stochasticity of $K(\mathbf{x})$ and thus $\mathbf{u}(\mathbf{x})$ renders these observables as stochastic processes. As we have seen in the previous section, the relative and absolute center of mass velocities are equal, $v_{i}(t)=v_{i}^{a}(t)=v_{i}^{r}(t)$. Furthermore, we find that the ensemble averages over $v_{i}(t)$ and the local center of mass velocity $\hat{v}_{i}\left(t \mid \mathbf{x}^{\prime}\right)$ (see equation (27)) are equal as well, and given by the mean flow velocity [Gelhar and Axness, 1983],

$$
\overline{v_{i}(t)}=\overline{\hat{v}_{i}\left(t \mid \mathbf{x}^{\prime}\right)}=\bar{u} \delta_{i 1},
$$

see also Appendix A. As an immediate consequence, we obtain from (43) that the ensemble averages of the absolute and relative dispersion coefficients are identical. It is denoted by $\bar{D}_{i j}^{r}(t)$ in the following equation:

$$
\bar{D}_{i j}^{r}(t)=\overline{D_{i j}^{a}(t)}=\overline{D_{i j}^{r}(t)} .
$$

[48] The ensemble average over the effective dispersion coefficient (28), $\overline{D_{i j}^{e}\left(t \mid \mathbf{x}^{\prime}\right)}$ for a plume evolving from a point-like injection at $\mathbf{x}^{\prime}$ is independent of the injection point due to the stationarity of the random flow field. As a direct consequence, we obtain from the right side of (30) that the ensemble average over $D_{i j}^{e}(t)$ is identical to the one for a point source. It is denoted in the following equation by

$$
\bar{D}_{i j}^{e}(t)=\overline{D_{i j}^{e}(t)}=\overline{D_{i j}^{e}\left(t \mid \mathbf{x}^{\prime}\right)}
$$

[49] The evolution of $\bar{D}_{i j}^{e}(t)$ and $\bar{D}_{i j}^{r}(t)$ has been studied in detail by Dentz et al. [2000a, 2000b]. Note that in Dentz et al. [2000b], $\bar{D}_{i j}^{r}(t)$ is referred to as effective dispersion coefficient for an extended source distribution.

[50] Now, we focus on quantifying the fluctuations of these observables about their respective ensemble averages. The variance of the center of mass velocity is given by

$$
\overline{\delta v_{i}(t)^{2}}=\overline{\left[v_{i}(t)-\bar{u} \delta_{i 1}\right]^{2}} .
$$

[51] The variance of the effective dispersion coefficient about its ensemble mean is

$$
\overline{\delta D_{i j}^{e}(t)^{2}}=\overline{\left[D_{i j}^{e}(t)-\bar{D}_{i j}^{e}(t)\right]^{2}}
$$

[52] We furthermore study the variances of the relative and absolute dispersion coefficients, which are given by

$$
\begin{aligned}
& \overline{\delta D_{i j}^{r}(t)^{2}}=\overline{\left[D_{i j}^{r}(t)-\bar{D}_{i j}^{r}(t)\right]^{2}} \\
& \overline{\delta D_{i j}^{a}(t)^{2}}=\overline{\left[D_{i j}^{a}(t)-\bar{D}_{i j}^{r}(t)\right]^{2}} .
\end{aligned}
$$

[53] As outlined earlier, the ensemble averages of the relative and absolute dispersion coefficients are the same, they differ, however, in the single realizations.

[54] The dispersion variances give valuable information about the self-averaging properties of mean velocity and the effective dispersion coefficients; this means that they quantify how well these quantities describe the actual dispersion behavior in a single disorder realization. At the same time, they provide information on the uncertainty of the dispersion behavior to be expected in a single realization with respect to the ensemble mean. Notice that for an extended source distribution, the effective dispersion coefficient (30) can be seen as spatial averages of the local effective dispersion coefficients over the initial distribution. In this sense, the variance is a measure for ergodicity of the dispersion processes as it compares spatial and ensemble average.

\section{Perturbation Theory}

[55] The variance of the center of mass velocity and dispersion coefficients (48) and (19)-(51) are determined using perturbation expansions in the fluctuations of the random field $\mathbf{u}(\mathbf{x})$. Here we establish the perturbation series for $c(\mathbf{x}, t)$ [e.g., Attinger et al., 1999; Dentz et al., 2000a]. 
[56] We start by inserting the decomposition $\mathbf{u}(\mathbf{x})=$ $\bar{u} \mathbf{e}_{1}+\mathbf{u}^{\prime}(\mathbf{x})$ into equation (2)

$$
\frac{\partial c(\mathbf{x}, t)}{\partial t}+\bar{u} \frac{\partial c(\mathbf{x}, t)}{\partial x_{1}}-\nabla \cdot[\mathbf{D} \nabla c(\mathbf{x}, t)]=-\mathbf{u}^{\prime}(\mathbf{x}) \cdot \nabla c(\mathbf{x}, t) .
$$

[57] For technical convenience, we perform a Fourier transform, which gives the integro-differential equation

$\frac{\partial \tilde{c}(\mathbf{k}, t)}{\partial t}-i \bar{u} k_{1} \tilde{c}(\mathbf{k}, t)+\mathbf{k} \cdot \mathbf{D k} c(\mathbf{k}, t)=i \mathbf{k} \cdot \int_{k^{\prime}} \tilde{\mathbf{u}}^{\prime}\left(\mathbf{k}^{\prime}\right) \tilde{c}\left(\mathbf{k}-\mathbf{k}^{\prime}, t\right)$.

[58] The latter can be integrated in time to give the equivalent integral equation

$$
\begin{aligned}
\tilde{c}(\mathbf{k}, t)= & \tilde{g}_{0}(\mathbf{k}, t) \tilde{\rho}(\mathbf{k})+\int_{0}^{t} \mathrm{~d} t^{\prime} \tilde{g}_{0}\left(\mathbf{k}, t-t^{\prime}\right) i \mathbf{k} \\
& \cdot \int_{k^{\prime}} \tilde{\mathbf{u}}^{\prime}\left(\mathbf{k}^{\prime}\right) \tilde{c}\left(\mathbf{k}-\mathbf{k}^{\prime}, t^{\prime}\right),
\end{aligned}
$$

where $\tilde{g}_{0}(\mathbf{k}, t)$ is given by

$$
\tilde{g}_{0}(\mathbf{k}, t)=\exp \left(-\mathbf{k} \cdot \mathbf{D k} t+i \bar{u} k_{1} t\right)
$$

[59] The Fourier transform of the initial condition (3) is given by

$$
\tilde{\rho}(\mathbf{k})=\prod_{i=1}^{d} \exp \left(-\frac{k_{i}^{2} L_{i}^{2}}{2}\right),
$$

and the Fourier transform of the correlation function (9) is

$$
\tilde{C}(\mathbf{k})=\sigma^{2}(2 \pi)^{d / 2} \prod_{i=1}^{d} l_{i} \exp \left(-\frac{k_{i}^{2} l_{i}^{2}}{2}\right) .
$$

[60] Note that the initial condition for the Fouriertransformed Green's function $\tilde{g}\left(\mathbf{k}, t \mid \mathbf{x}^{\prime}\right)$ is given by

$$
\tilde{\rho}(\mathbf{k})=\exp \left(i \mathbf{k} \cdot \mathbf{x}^{\prime}\right) .
$$

[61] We establish a perturbation series in the fluctuations $\tilde{\mathbf{u}}^{\prime}(\mathbf{k})$ by iteration of the integral equation (54)

$$
\tilde{c}(\mathbf{k}, t)=\tilde{c}_{0}(\mathbf{k}, t)+\tilde{c}_{1}(\mathbf{k}, t)+\tilde{c}_{2}(\mathbf{k}, t)+\cdots,
$$

where the zeroth-, first-, and second-order contributions are given by

$$
\begin{gathered}
\tilde{c}_{0}(\mathbf{k}, t)=\tilde{g}_{0}(\mathbf{k}, t) \tilde{\rho}(\mathbf{k}) \\
\tilde{c}_{1}(\mathbf{k}, t)=\int_{0}^{t} \mathrm{~d} t^{\prime} \tilde{g}_{0}\left(\mathbf{k}, t-t^{\prime}\right) \int_{k^{\prime}} i \mathbf{k} \cdot \tilde{\mathbf{u}}^{\prime}\left(\mathbf{k}^{\prime}\right) \tilde{c}_{0}\left(\mathbf{k}-\mathbf{k}^{\prime}, t^{\prime}\right)
\end{gathered}
$$

$$
\begin{aligned}
\tilde{c}_{2}(\mathbf{k}, t)= & \int_{0}^{t} \mathrm{~d} t^{\prime} \tilde{g}_{0}\left(\mathbf{k}, t-t^{\prime}\right) \int_{k^{\prime}} i \mathbf{k} \cdot \tilde{\mathbf{u}}^{\prime}\left(\mathbf{k}^{\prime}\right) \int_{0}^{t^{\prime}} \mathrm{d} \mathbf{t}^{\prime \prime} \tilde{g}_{0}\left(\mathbf{k}-\mathbf{k}^{\prime}, t^{\prime}-\mathbf{t}^{\prime \prime}\right) \\
& \times \int_{k^{\prime \prime}} i\left(\mathbf{k}-\mathbf{k}^{\prime}\right) \cdot \tilde{\mathbf{u}}^{\prime}\left(\mathbf{k}^{\prime \prime}\right) \tilde{c}_{0}\left(\mathbf{k}-\mathbf{k}^{\prime}-\mathbf{k}^{\prime \prime}, \mathbf{t}^{\prime \prime}\right) .
\end{aligned}
$$

[62] Note that the perturbation series for the Green's function $\tilde{g}\left(\mathbf{k}, t \mid \mathbf{x}^{\prime}\right)$ is obtained by inserting (58) for $\rho(\mathbf{k})$ in the earlier expressions.

[63] Inserting (59) for $\tilde{c}(\mathbf{k}, t)$ and $\tilde{g}\left(\mathbf{k}, t \mid \mathbf{x}^{\prime}\right)$, respectively, into expressions (21), (31), and (38) for the generating functions gives the analogous expansions

$$
\begin{gathered}
\chi^{\gamma}(\mathbf{k}, t)=\chi_{0}^{\gamma}(\mathbf{k}, t)+\chi_{1}^{\gamma}(\mathbf{k}, t)+\chi_{2}^{\gamma}(\mathbf{k}, t)+\cdots \\
\hat{\chi}\left(\mathbf{k}, t \mid \mathbf{x}^{\prime}\right)=\chi_{0}^{r}\left(\mathbf{k}, t \mid \mathbf{x}^{\prime}\right)+\hat{\chi}_{1}\left(\mathbf{k}, t \mid \mathbf{x}^{\prime}\right)+\hat{\chi}_{2}\left(\mathbf{k}, t \mid \mathbf{x}^{\prime}\right)+\cdots,
\end{gathered}
$$

where $\gamma=a, r$. Inserting these expressions into (22), (23), (32), and (39) and subsequent expansion up to second order gives the corresponding perturbation expansions for the center of mass velocity and dispersion coefficients

$$
\begin{array}{r}
v_{i}(t)=\bar{u} \delta_{i 1}+\delta^{(1)}\left\{v_{i}(t)\right\}+\delta^{(2)}\left\{v_{i}(t)\right\}+\cdots \\
D_{i j}^{\alpha}(t)=D_{i j}+\delta^{(1)}\left\{D_{i j}^{\alpha}(t)\right\}+\delta^{(2)}\left\{D_{i j}^{\alpha}(t)\right\}+\cdots,
\end{array}
$$

where the superscript $\alpha=a, e, r$. The terms denoted by $\delta^{(i)}\{\cdot\}$ are of $i$ th order in the fluctuations of the random velocity field. Inserting (65) into (48), we obtain for the variance of the center of mass velocity up to second order in the fluctuations of the random velocity field

$$
\overline{\delta v_{i}(t)^{2}}=\overline{\delta^{(1)}\left\{v_{i}(t)\right\}^{2}}+\cdots,
$$

[64] The variance of the dispersion coefficients of the absolute, effective, and relative dispersion coefficients is obtained by inserting (66) into (49)-(51) and yields

$$
\overline{\delta D_{i j}^{\alpha}(t)^{2}}=\overline{\delta^{(1)}\left\{D_{i j}^{\alpha}(t)\right\}^{2}}+\cdots
$$

[65] Thus, the second-order contributions to the fluctuation variances depend only on the respective first-order contributions. We obtain $\delta^{(1)}\left\{v_{i}(t)\right\}$ and $\delta^{(1)}\left\{D_{i j}^{\alpha}(t)\right\}$, with $\alpha=a, e, r$ by inserting the perturbation expansion (59) into the definitions (22) and (23), (32) and (39), and subsequent expansion of the resulting expressions. This gives

$$
\begin{gathered}
\delta^{(1)}\left\{v_{i}(t)\right\}=-\left.i \frac{\mathrm{d}}{\mathrm{d} t} \frac{\partial}{\partial k_{i}} \frac{\chi_{1}^{a}(\mathbf{k}, t)}{\chi_{0}^{a}(\mathbf{k}, t)}\right|_{\mathbf{k}=\mathbf{0}} \\
\delta^{(1)}\left\{D_{i j}^{\gamma}(t)\right\}=-\left.\frac{1}{2} \frac{\mathrm{d}}{\mathrm{d} t} \frac{\partial^{2}}{\partial k_{i} \partial k_{j}} \frac{\chi_{1}^{\gamma}(\mathbf{k}, t)}{\chi_{0}^{\gamma}(\mathbf{k}, t)}\right|_{\mathbf{k}=\mathbf{0}} . \\
\delta^{(1)}\left\{D_{i j}^{e}(t)\right\}=-\left.\frac{1}{2} \frac{\mathrm{d}}{\mathrm{d} t} \int \mathrm{d} \mathbf{x}^{\prime} \rho\left(\mathbf{x}^{\prime}\right) \frac{\partial^{2}}{\partial k_{i} \partial k_{j}} \frac{\hat{\chi}_{1}\left(\mathbf{k}, t \mid \mathbf{x}^{\prime}\right)}{\hat{\chi}_{0}\left(\mathbf{k}, t \mid \mathbf{x}^{\prime}\right)}\right|_{\mathbf{k}=\mathbf{0}} .
\end{gathered}
$$

for $\gamma=a, r$. 
[66] The generating function $\chi^{a}(\mathbf{k}, t)$ (equation (21)) is by definition equal to the Fourier transform of the concentration distribution $\tilde{c}(\mathbf{k}, t)$, and therefore, $\chi_{0}^{a}(\mathbf{k}, t)$ and $\chi_{1}^{a}(\mathbf{k}, t)$ are given by (60) and (61),

$$
\chi_{0}^{a}(\mathbf{k}, t)=\tilde{c}_{0}(\mathbf{k}, t), \quad \chi_{1}^{a}(\mathbf{k}, t)=\tilde{c}_{1}(\mathbf{k}, t) .
$$

[67] For the generating function $\hat{\chi}\left(\mathbf{k}, t \mid \mathbf{x}^{\prime}\right)$, we obtain by inserting (60) and (61) into (31)

$$
\begin{gathered}
\hat{\chi}_{0}\left(\mathbf{k}, t \mid \mathbf{x}^{\prime}\right)=\tilde{g}_{0}(\mathbf{k}, t) \\
\hat{\chi}_{1}\left(\mathbf{k}, t \mid \mathbf{x}^{\prime}\right)=\int_{0}^{t} \mathrm{~d} t^{\prime} \tilde{g}_{0}\left(\mathbf{k}, t-t^{\prime}\right) \int_{k^{\prime}} i \mathbf{k} \\
\cdot \tilde{\mathbf{u}}^{\prime}\left(\mathbf{k}^{\prime}\right) \tilde{g}_{0}\left(\mathbf{k}-\mathbf{k}^{\prime}, t^{\prime}\right) \exp \left(-i \mathbf{k}^{\prime} \cdot \mathbf{x}^{\prime}\right) .
\end{gathered}
$$

[68] Analogously, we obtain for $\chi^{r}(\mathbf{k}, t)$

$$
\begin{gathered}
\chi_{0}^{r}(\mathbf{k}, t)=\tilde{g}_{0}(\mathbf{k}, t) \\
\chi_{1}^{r}(\mathbf{k}, t)=\int_{0}^{t} \mathrm{~d} t^{\prime} \tilde{g}_{0}\left(\mathbf{k}, t-t^{\prime}\right) \int_{k^{\prime}} i \mathbf{k} \cdot \tilde{\mathbf{u}}^{\prime}\left(\mathbf{k}^{\prime}\right) \tilde{g}_{0}\left(\mathbf{k}-\mathbf{k}^{\prime}, t^{\prime}\right) \rho\left(-\mathbf{k}^{\prime}\right) .
\end{gathered}
$$

[69] Inserting (75) and (76) into (70) for $\gamma=r$, and (73) and (74) into (71), we find that the first-order contributions to the global effective and the relative dispersion coefficients are in fact equal. Thus, according to (68), also the respective variances are equal in this approximation

$$
\overline{\delta D_{i j}^{r}(t)^{2}}=\overline{\delta D_{i j}^{e}(t)^{2}} \text {. }
$$

[70] Notice that the two dispersion quantities in general differ in individual realizations, and also their ensemble averages are different. However, as their respective firstorder approximations are equal, as pointed out earlier, we find that for up to second-order in the fluctuations of the random flow field, their variances with respect to the respective ensemble averages are identical. Thus, in the following, we will focus on the fluctuation behavior of the effective and the absolute dispersion coefficients.

\section{Explicit Expressions}

[71] We derive explicit perturbation expressions for the variances of the center of mass velocity and dispersion coefficients in order to study the fluctuation behavior of transport in heterogeneous flow fields.

\subsection{Center of Mass Velocity}

[72] Inserting (60) and (61) into (69) and using (56), after some algebra one obtains

$$
\delta^{(1)}\left\{v_{i}(t)\right\}=\int_{k} \tilde{g}_{0}(-\mathbf{k}, t) \tilde{\rho}(\mathbf{k}) \tilde{u}_{i}{ }^{\prime}(\mathbf{k}) .
$$

[73] Thus, we obtain for the variance of the center of mass velocity the integral expression

$$
\overline{\delta v_{i}^{2}(t)}=\bar{u}^{2} \int_{k} \tilde{g}_{0}(-\mathbf{k}, t) \tilde{g}_{0}(\mathbf{k}, t) \tilde{\rho}(\mathbf{k}) \tilde{\rho}(-\mathbf{k}) p_{i}(\mathbf{k})^{2} \tilde{C}(\mathbf{k})
$$

[74] By inserting expressions (55)-(57) for the propagator $\tilde{g}_{0}(\mathbf{k}, t)$, the initial distribution $\tilde{\rho}(\mathbf{k})$, and the correlation function $\widetilde{C}(\mathbf{k})$, respectively, we obtain

$$
\overline{\delta v_{i}^{2}(t)}=\sigma^{2} \bar{u}^{2}(2 \pi)^{d / 2} \int_{k} p_{i}(\mathbf{k})^{2} \prod_{n=1}^{d} l_{n} \exp \left[-\frac{k_{n}^{2} l_{n}^{2} B_{n}(t)^{2}}{2}\right],
$$

where we define

$$
B_{n}(t)=\sqrt{1+2 \xi_{n}^{2}+\frac{4 t}{\tau_{D_{n}}}}, \xi_{n}^{2}=\frac{L_{n}^{2}}{l_{n}^{2}} .
$$

[75] Recall that the dispersion time scales $\tau_{D_{n}}=l_{n}^{2} / D_{n}$ quantify the typical time needed by the solute to cover the distance of a correlation length by local dispersion.

[76] Expression (80) can be evaluated explicitly for the fully anisotropic scenario in $d=2$ spatial dimensions. The corresponding expressions are given in Appendix B. In the fully isotropic case of $l_{i}=l, D_{i}=D$, and $L_{i}=L$ for $i=1,2$, we find $\overline{\delta v_{2}^{2}(t)}=\overline{\delta v_{1}^{2}(t)} / 3$, with

$$
\overline{\delta v_{1}^{2}(t)}=\frac{3 \sigma^{2} \bar{u}^{2}}{8\left(1+2 \xi+4 t / \tau_{D}\right)^{2}} .
$$

[77] For $d=3$ spatial dimensions, there is no simple closed form solution for the fully anisotropic case. Integral expressions are given in Appendix B. In the fully isotropic case, we obtain $\overline{\delta v_{2}^{2}(t)}=\overline{\delta v_{3}^{2}(t)}=1 / 8 \overline{\delta v_{1}^{2}(t)}$ with

$$
\overline{\delta v_{1}^{2}(t)}=\frac{8 \sigma^{2} \bar{u}^{2}}{15\left(1+2 \xi+4 t / \tau_{D}\right)^{3}} .
$$

\subsection{Dispersion Coefficients}

[78] The first-order contribution to the absolute dispersion coefficients is obtained by inserting (60) and (61) into (70) and using the explicit expression (56) for the initial solute distribution. For the first-order contribution to the effective dispersion coefficient, we insert (75) and (76) in (70) together with (55). This gives

$$
\begin{aligned}
\delta^{(1)}\left\{D_{i j}^{\gamma}(t)\right\}= & -\frac{1}{2} \int_{k} \tilde{g}_{0}(-\mathbf{k}, t) \tilde{\rho}(-\mathbf{k}) i \\
& {\left[\tilde{u}_{i}^{\prime}(\mathbf{k}) k_{j} A_{j}^{\gamma}(t)^{2} l_{j}^{2}+\tilde{u}_{j}^{\prime}(\mathbf{k}) k_{i} A_{i}^{\gamma}(t)^{2} l_{i}^{2}\right], }
\end{aligned}
$$

with $\gamma=a, e$. The auxiliary functions are defined

$$
A_{i}^{a}(t)=\sqrt{\xi_{i}^{2}+\frac{2 t}{\tau_{D_{i}}}}, \quad A_{i}^{e}(t)=\sqrt{\frac{2 t}{\tau_{D_{i}}}} .
$$

[79] Recall that $\xi_{i}=L_{i} / l_{i}$. Thus, we obtain for the variance of the apparent dispersion coefficients the perturbation theory expression 


$$
\begin{aligned}
\overline{\delta D_{i j}^{\gamma}(t)^{2}}= & \frac{1}{4} \int_{k} \tilde{g}_{0}(-\mathbf{k}, t) \tilde{g}_{0}(\mathbf{k}, t) \tilde{\rho}(\mathbf{k}) \tilde{\rho}(-\mathbf{k}) \tilde{C}(\mathbf{k}) \\
& \times\left[p_{i}(\mathbf{k})^{2} k_{j}^{2} A_{j}^{\gamma}(t)^{4} l_{j}^{4}+p_{j}(\mathbf{k})^{2} k_{i}^{2} A_{i}^{\gamma}(t)^{4} l_{i}^{4}\right. \\
& \left.+2 p_{i}(\mathbf{k}) p_{j}(\mathbf{k}) k_{i} k_{j} A_{i}^{\gamma}(t)^{2} A_{j}^{\gamma}(t)^{2} l_{i}^{2} l_{j}^{2}\right]
\end{aligned}
$$

[80] In the following, we evaluate these expression for $d=2$ and $d=3$ spatial dimensions.

\subsubsection{Two Spatial Dimensions}

[81] For $d=2$ spatial dimensions, these expressions can be evaluated explicitly and are given in Appendix. For the completely isotropic case of $l_{i}=l, D_{i}=D$, and $L_{i}=L$ for $i=1,2$, we obtain for the variance of the absolute dispersion coefficients the simple expressions

$$
\overline{\delta D_{i j}^{a}(t)^{2}}=\frac{1}{8} \sigma^{2} \bar{u}^{2} l^{2}\left(\frac{\xi^{2}+2 t / \tau_{D}}{1+2 \xi^{2}+4 t / \tau_{D}}\right)^{2},
$$

for $i, j=1,2$. For the variance of the effective dispersion coefficient, we obtain accordingly

$$
\overline{\delta D_{i j}^{e}(t)^{2}}=\frac{1}{8} \sigma^{2} \bar{u}^{2} l^{2}\left(\frac{2 t / \tau_{D}}{1+2 \xi^{2}+4 t / \tau_{D}}\right)^{2} .
$$

[82] This means that in the isotropic case, the variances of the diagonal and off-diagonal dispersion coefficients are identical. This is a noteworthy result because the respective ensemble averages are very different from each other [e.g., Dentz et al., 2000a].

[83] In the asymptotic limit of infinite times, $t \rightarrow \infty$, the variances of the absolute and effective coefficients converge to the same constant value

$$
\lim _{t \rightarrow \infty} \overline{\delta D_{i j}^{a}(t)^{2}}=\lim _{t \rightarrow \infty} \overline{\delta D_{i j}^{e}(t)^{2}}=\frac{1}{32} \sigma^{2} \bar{u}^{2} l^{2}
$$

[84] This means that both the absolute and effective dispersion coefficients are not self-averaging observables because the variance of their sample-to-sample fluctuations assumes a constant value in the long time limit. In the limit of small times, $t \ll \tau_{D}$, the variances of the absolute and effective variances behave very differently. The variance of the absolute dispersion coefficients starts from a finite value at $t=0$,

$$
\lim _{t \rightarrow 0} \overline{\delta D_{i j}^{a}(t)^{2}}=\frac{1}{8} \sigma^{2} \bar{u}^{2} l^{2} \frac{\xi^{4}}{\left(1+2 \xi^{2}\right)^{2}},
$$

which implies that the absolute dispersion coefficients are uncertain from the beginning. This uncertainty can be traced back to the cross terms between initial position and flow velocity that contribute to $D_{i j}^{a}(t)$ (see equation (43)). The variance of the effective dispersion coefficients, in contrast, is going to zero as time goes to zero, $\lim _{t \rightarrow 0} \overline{\delta D_{i j}^{e}(t)^{2}}=0$, and thus is initially not subject to uncertainty. This is so because the effective, as well as the relative, dispersion coefficients are initially given by the local dispersion coefficients, $D_{i j}^{e}(0)=D_{i j}^{a}(0)=D_{i j}$.

\subsubsection{Three Spatial Dimensions}

[85] For $d=3$ dimensions, the expressions for the fully anisotropic problem are given in Appendix C. For the isotropic case of $D_{i}=D, l_{i}=l$, and $L_{i}=L$ for $i=1,2,3$, we find

$$
\overline{\delta D_{i j}^{\gamma}(t)^{2}}=b_{i j} \overline{\delta D_{11}^{\gamma}(t)^{2}},
$$

where $\quad b_{22}=b_{33}=3 / 8, \quad b_{12}=b_{13}=19 / 32, \quad$ and $b_{23}=1 / 8$, with $\gamma=a, e$. For $\overline{\delta D_{11}^{a}(t)^{2}}$, we obtain the compact expression

$$
\overline{\delta D_{11}^{a}(t)^{2}}=\frac{8}{35} \sigma^{2} \bar{u}^{2} l^{2} \frac{\left(\xi^{2}+2 t / \tau_{D}\right)^{2}}{\left(1+2 \xi^{2}+4 t / \tau_{D}\right)^{5 / 2}},
$$

and for $\overline{\delta D_{11}^{e}(t)^{2}}$ correspondingly

$$
\overline{\delta D_{11}^{e}(t)^{2}}=\frac{8}{35} \sigma^{2} \bar{u}^{2} l^{2} \frac{\left(2 t / \tau_{D}\right)^{2}}{\left(1+2 \xi^{2}+4 t / \tau_{D}\right)^{5 / 2}} .
$$

[86] Unlike for $d=2$ dimensions, the dispersion coefficients here are self-averaging in the long time limit. For times much larger than the dispersion time scale $\tau_{D}=l^{2} / D$ the variances of both the absolute and effective dispersion coefficients tend to zero as $t^{-1 / 2}$. At small times, we find the same discrepancy between the behaviors of the absolute and effective coefficients as in $d=2$ spatial dimensions. While $\overline{\delta D_{11}^{a}(t)^{2}}$ tends to the finite value

$$
\lim _{t \rightarrow 0} \overline{\delta D_{11}^{a}(t)^{2}}=\frac{8}{35} \sigma^{2} \bar{u}^{2} l^{2} \frac{\xi^{4}}{\left(1+2 \xi^{2}\right)^{5 / 2}}
$$

the variance $\overline{\delta D_{11}^{e}(t)^{2}}$ of effective and relative dispersion coefficients is zero for $t=0$.

\section{Results and Discussion}

[87] In this section, we discuss the evolution of the variances of the respective dispersion coefficients in $d=2$ and $d=3$ dimensions for different initial plume dimensions and geometries measured through the relative source extension $\xi_{i}=L_{i} / l_{i}$. For clarity, we assume isotropic localscale dispersion, $D_{i i}=D$, and statistic isotropy so that the correlation lengths $l_{i}=l$ for $i=1, \cdots, d$. This implies that the dispersion time scales are $\tau_{D_{i}}=\tau_{D}=l^{2} / D$, and the Péclet number is given by $P e_{i}=P e=\bar{u} l / D$. All the results for the dispersion variances shown in the following are normalized by $\sigma^{2} \bar{u}^{2} l^{2}$, and time is normalized by the advection time scale $\tau_{u}$.

\subsection{Two Spatial Dimensions}

[88] For a point source, the absolute, effective, and relative dispersion coefficients, and therefore also their respective variances, are identical, as pointed out in section 3.4. Figure 1 displays the time evolution of the isotropic dispersion variance for a point source, given by setting $\xi=0$ in (87) or (88). As shown in Figure 1, for times smaller than 


\section{$\overline{\delta \mathrm{D}(t)^{2}}$}

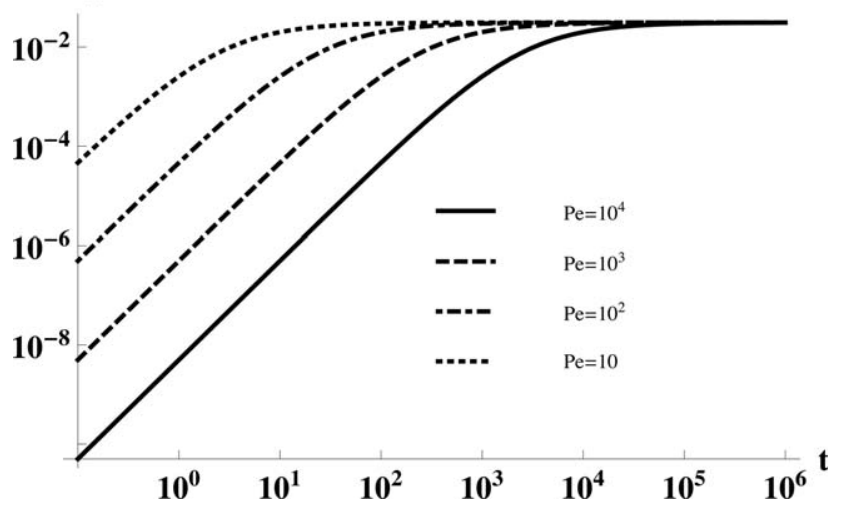

Figure 1. Variances of the dispersion coefficients for a point-like injection. Note that here the $\overline{\delta D_{i j}^{\gamma}(t)^{2}}=\overline{\delta D(t)^{2}}$ with $\gamma=a, e$ is independent of the direction and equal for absolute and effective dispersion.

the dispersion time scale, $t \ll \tau_{D}$, the variance $\overline{\delta D_{11}^{e}(t)^{2}}$ evolves as $\left(t / \tau_{D}\right)^{2}$ and converges toward its asymptotic long time value on the dispersion time scale $\tau_{D}$. In the limit of $P e \rightarrow \infty$, the variance $\overline{\delta D_{11}^{e}(t)^{2}} \rightarrow 0$ (see equation (88)). In this case, this means that for purely advective transport, the solute will travel and meander along the streamline on which it was released, and dispersion is zero. For finite $P e$, the solute samples neighboring streamlines leading to increasing dispersive fluctuations. Notice that the behavior displayed in Figure 1 differs from the one reported by Eberhard et al. [2007] for the coefficient of variation of the longitudinal dispersion coefficient. This discrepancy may be traced back to the Lagrangian iteration method that has been used by these authors to numerically approximate the dispersion coefficients in single disorder realizations.

[89] In the following figures, we set $P e=10^{3}$. Figure 2 illustrates the time behavior of the absolute and effective variances for different source dimensions given by (87) and (88), respectively. The time evolution of $\overline{\delta D_{11}^{a}(t)^{2}}$ is shown in Figure 2a. It starts from the finite initial value (90), which increases with increasing source size. For $\xi^{2} \tau_{D} \ll t \ll \tau_{D}$, the time behavior is similar to the one observed for the point source. This can be observed for $\xi=10^{-1}$. For increasing source size, the initial value $\overline{\delta D_{11}^{a}(0)^{2}}$ converges toward the asymptotic long time value,

$$
\lim _{\xi \rightarrow \infty} \overline{\delta D_{11}^{a}(0)^{2}}=\lim _{t \rightarrow \infty} \overline{\delta D_{11}^{a}(t)^{2}} .
$$

[90] Notice that the initial and asymptotic values of $\overline{\delta D_{11}^{a}(t)^{2}}$ are related by

$$
\lim _{t \rightarrow \infty} \overline{\delta D_{11}^{a}(t)^{2}}=\left[1+\frac{1}{2 \xi^{2}}\right]^{2} \overline{\delta D_{11}^{a}(0)^{2}},
$$

compare (89) and (90). Thus, for large $\xi$ the variance appears constant because its range of variability is $\overline{\delta D_{11}^{a}(0)^{2}} /\left(4 \xi^{4}\right)$. (a)

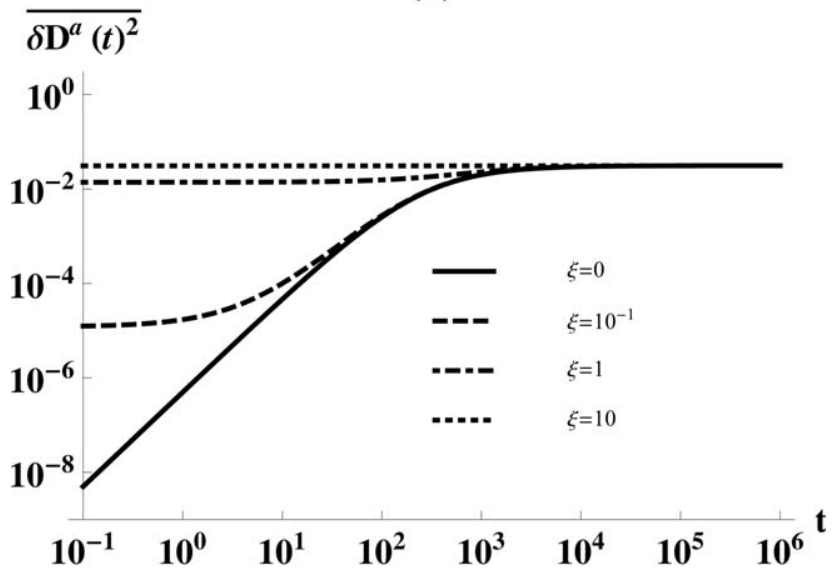

(b)

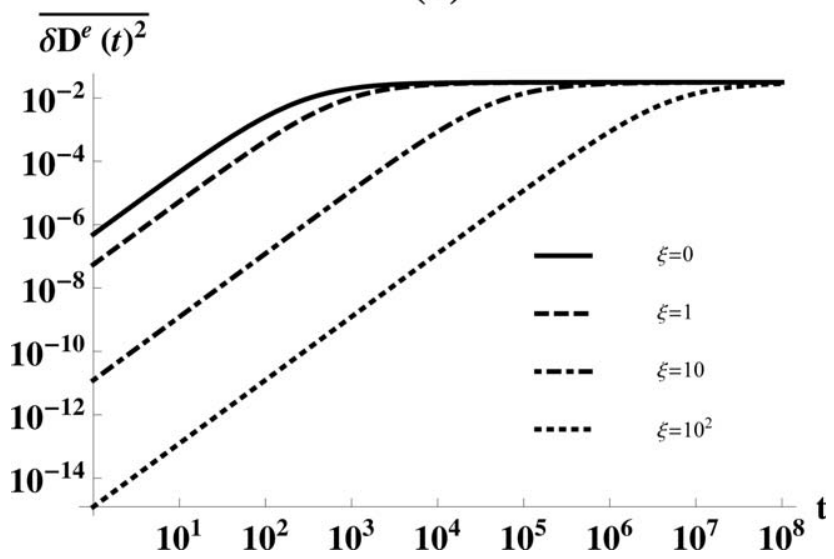

Figure 2. Variances of the (a) absolute and (b) effective dispersion coefficients for an isotropic source distribution of dimensions $\xi=0,10^{-1}, 1,10$ and $\xi=0,1,10,10^{2}$. Note that here the $\overline{\delta D_{i j}^{\gamma}(t)^{2}}=\overline{\delta D^{\gamma}(t)^{2}}$ is independent of the direction, see (87) and (88) for $\gamma=a, e$.

[91] Notice that the increase of uncertainty with increasing source size at preasymptotic times is counterintuitive. One would expect that the system is more self-averaging because the solute samples more heterogeneity from the beginning. As previously discussed, however, this initial uncertainty is due to correlations between the initial particle position $\mathbf{x}^{\prime}$ and the mean velocity of particles originating from $\mathbf{x}^{\prime}$ (see (43) and (44)). This cross-correlation term was identified in the past by Sposito and Dagan [1994] and Fiori and Jankovic [2005] who investigated the convergence of the second central moments to their ergodic ensemble value [see also Suciu et al., 2008].

[92] The time evolution of the variance of the effective dispersion coefficients shown in Figure $2 \mathrm{~b}$ is more intuitive. By definition, $D_{i j}^{e}(0)=D_{i j}$, and therefore, the initial uncertainty is zero. The variance at preasymptotic times is decreasing for increasing source size reflecting the fact that the solute samples more of the medium heterogeneity from the beginning, which decreases the uncertainty. The time scale at which the variance becomes asymptotic is affected 
by the size of the initial plume. The variance approaches its constant asymptotic long time value (89) for times $t \gg \tau_{D}\left(1+2 \xi^{2}\right)$.

[93] We now consider the case of a line source perpendicular to the mean flow direction so that $\xi_{1}=0$. This implies that

$$
\overline{\delta D_{11}^{a}(t)^{2}}=\overline{\delta D_{11}^{e}(t)^{2}}
$$

which follows from (86) and $A_{i}^{\gamma}(t)$ given by (85). Figure 3 illustrates the time evolution of both the absolute and effective longitudinal dispersion variances for an extended transverse line source of varying $\xi_{2}$. At preasymptotic times, the uncertainty decreases with increasing source dimension, as observed in the previous section for the effective dispersion variance. Again, as the plume dimension increases, the solute samples more of the medium heterogeneity and the variance decreases. However, at asymptotically long times, all curves converge to the asymptotic value (89). The asymptotic time regime is defined here by $t \gg \tau_{D}\left(1+2 \xi_{2}^{2}\right)$.

[94] The time evolution of the variances of the transverse dispersion coefficients is shown in Figures $4 \mathrm{a}$ and $4 \mathrm{~b}$. Unlike for the variance of the longitudinal coefficients, the temporal behavior of the absolute and effective quantities is quite distinct, while at times $t \gg \tau_{D}\left(1+2 \xi_{2}^{2}\right)$ both converge to the asymptotic long time value (89).

[95] Figure 4a illustrates the time behavior of the absolute dispersion variance. It evolves from the finite initial value

$$
\overline{\delta D_{22}^{a}(0)^{2}}=\sigma^{2} \bar{u}^{2} l^{2} \frac{\xi_{2}^{4}}{\left(1+2 \xi_{2}^{2}\right)^{2}}
$$

which can be obtained by taking the limit $t \rightarrow 0$ in (C3). The initial uncertainty increases with increasing source size, but not unboundedly. For $\xi_{2} \rightarrow \infty$, it converges to the asymptotic value $\sigma^{2} \bar{u}^{2} l^{2} / 4$. As discussed in the previous

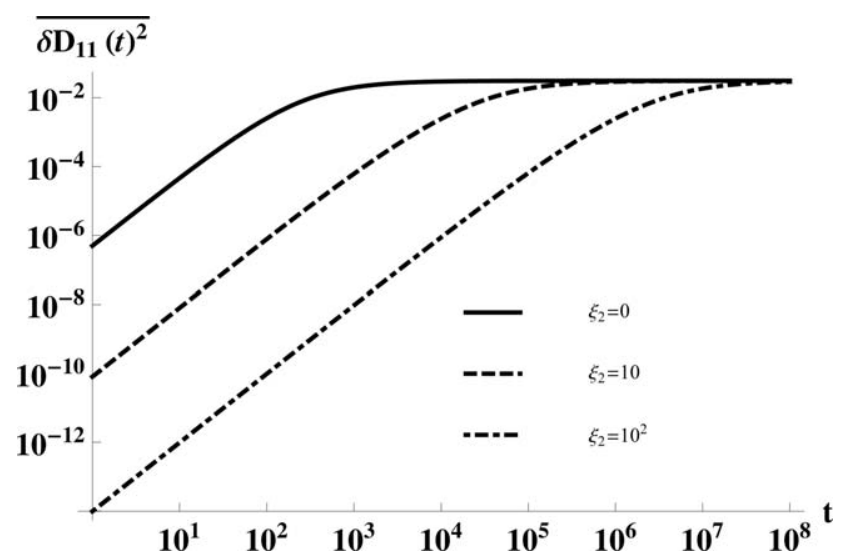

Figure 3. Variances of the longitudinal dispersion coefficients with $\xi_{1}=0$ and line sources orientated perpendicular to the mean flow directions with $\xi_{2}=0,10,10^{2}$ in $d=2$ spatial dimensions. Note that in this case $\overline{\delta D_{11}^{a}(t)^{2}}=\overline{\delta D_{11}^{e}(t)^{2}}=\overline{\delta D_{11}(t)^{2}}$. (a)

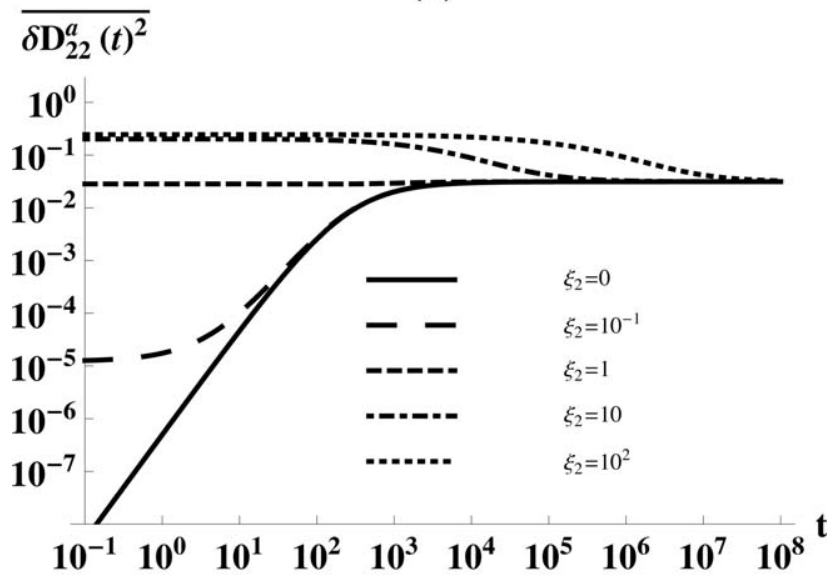

(b)

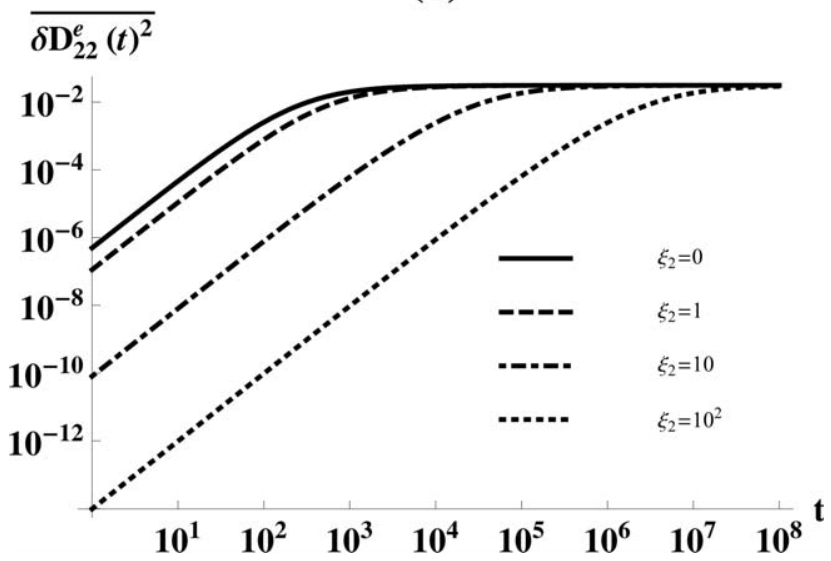

Figure 4. Variances of the longitudinal (a) absolute and (b) effective dispersion coefficients with $\xi_{1}=0$ and line sources orientated perpendicular to the mean flow directions with $\xi_{2}=0,10^{-1}, 1,10,10^{2}$ and $\xi_{2}=0,1,10,10^{2}$ in $d=2$ spatial dimensions.

section, this counterintuitive behavior is due to cross correlations between the fluctuations of the initial particle positions with respect to the initial center of mass of the plume and the initial particle velocities (see equation (44)). Figure $4 \mathrm{~b}$ shows the evolution of the effective dispersion variance. Its initial value is zero, and the preasymptotic uncertainty decreases with increasing $\xi_{2}$. As shown by Dagan [1991] and Zhang et al. [1996], the orientation of the line source has a strong impact on the quantification of the average spreading behavior. We now illustrate that the same holds for the variance. For a line source orientated along the mean flow direction, this means that $\xi_{2}=0$, the behavior is complementary to the one discussed earlier. Here the variances of the absolute and effective transverse variances behave in the same way, which again follows from (86) and definition (85) of the $A_{i}^{\gamma}(t)$, while the variances of the longitudinal coefficients show a different time behavior. These behaviors are illustrated in Figures $5 \mathrm{a}$ and $5 \mathrm{~b}$ and 6 for the variances of the longitudinal and transverse coefficients, respectively. The variance of the absolute longitudinal dispersion coefficient here has the finite initial value 
(a)

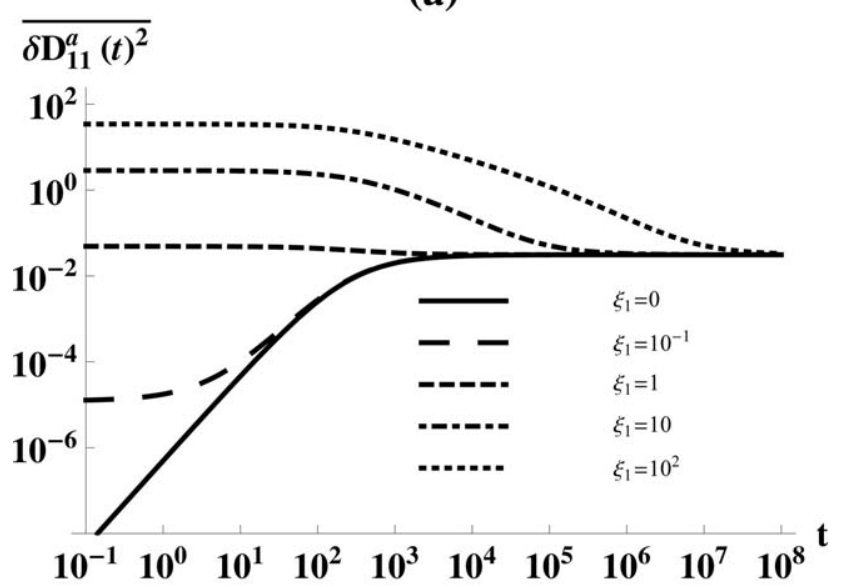

(b)

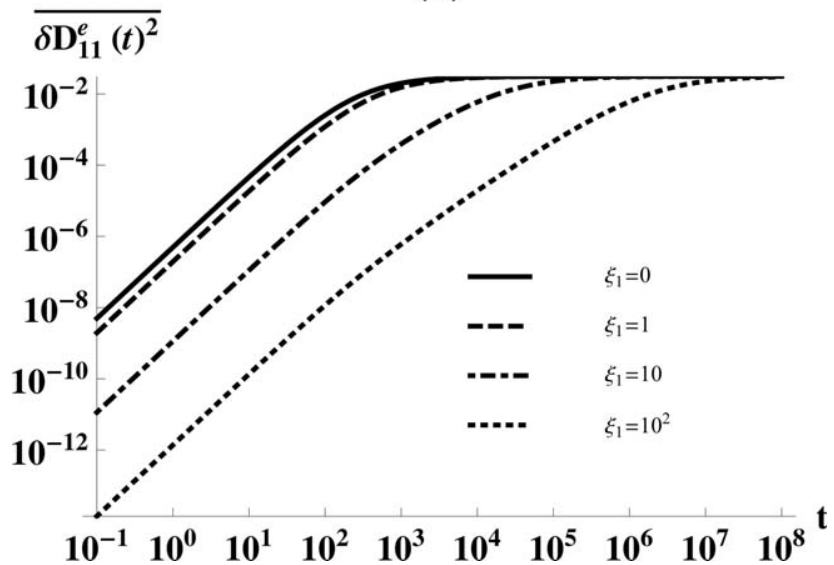

Figure 5. Variances of the longitudinal (a) absolute and (b) effective dispersion coefficients with $\xi_{2}=0$ and line sources orientated along the mean flow directions with $\xi_{1}=0,10^{-1}, 1,10,10^{2}$ and $\xi_{1}=0,1,10,10^{2}$ in $d=2 \mathrm{spa}-$ tial dimensions.

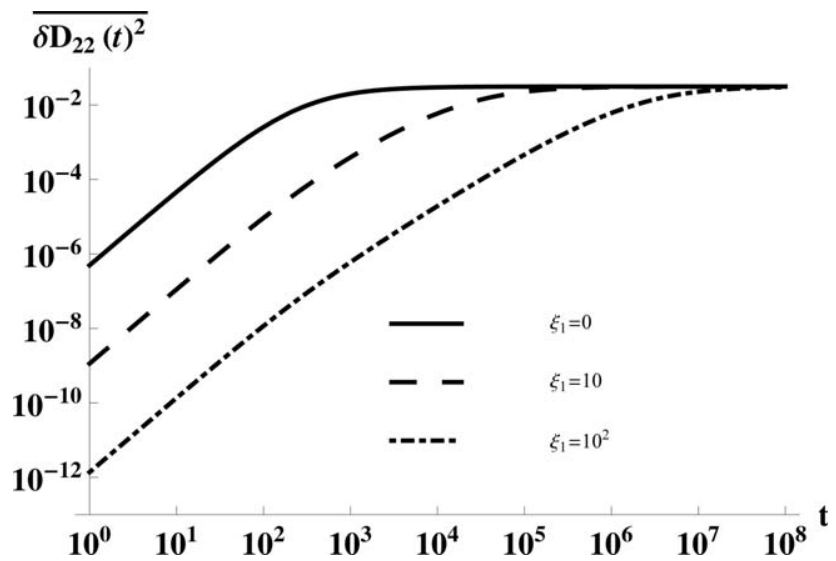

Figure 6. Variances of the transverse dispersion coefficients with $\xi_{2}=0$ and line sources orientated along the mean flow directions with $\xi_{1}=0,10,10^{2}$ in $d=2$ spatial dimensions. Note that in this case $\overline{\delta D_{22}^{a}(t)^{2}}=$ $\overline{\delta D_{22}^{e}(t)^{2}}=\overline{\delta D_{22}(t)^{2}}$.

$$
\overline{\delta D_{11}^{a}(0)^{2}}=\sigma^{2} \bar{u}^{2} l^{2} \frac{\xi_{1}^{4}}{\left(1+\sqrt{1+2 \xi_{1}^{2}}\right)^{3}} .
$$

[96] It is noteworthy that here unlike for the uncertainty of the transverse coefficients discussed previously, the variance increases monotonically with the source size as $\xi_{1}^{1 / 2}$.

\subsection{Three Spatial Dimensions}

[97] Figure 7 shows the time behavior of the absolute and effective dispersion coefficients for the fully isotropic scenario given by expressions (92) and (93) for $P e=10^{3}$. The evolution of the absolute dispersion variance is illustrated in Figure 7a for varying source sizes. As for $d=2$ dimensions, the initial uncertainty is nonzero for $\xi>0$ (see equation (94)), due to the correlation of initial particle positions and velocities. The initial value here is a nonmonotonic function of the initial plume size (see equation (94)). For initial plume sizes smaller than the correlation length, $\xi \leq 1$, the variance increases from its initial value toward a maximum, which is attained on the dispersion time scale $\tau_{D}$. It then decreases toward zero according to $t^{-1 / 2}$ (see equation (92)). For $\xi>10^{-1}$, the variance remains almost

(a)

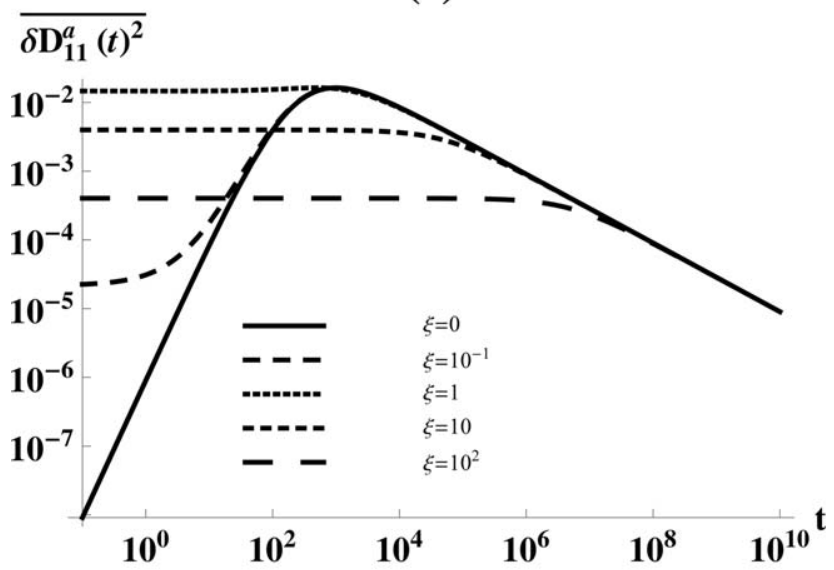

(b)

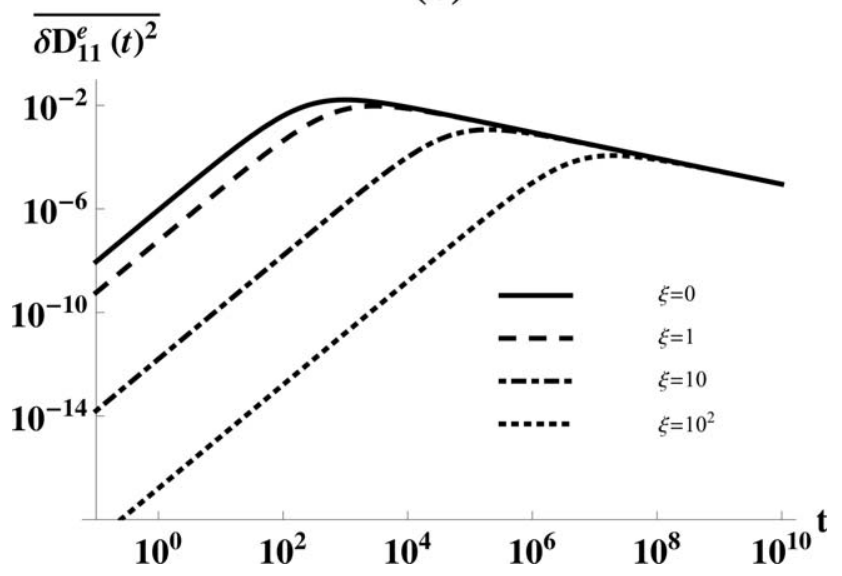

Figure 7. Variances of the (a) absolute and (b) effective dispersion coefficients for isotropic source distribution $\xi_{1}=\xi_{2}=\xi_{3}=\xi=0,10^{-1}, 1,10,10^{2}$ and $\xi_{1}=\xi_{2}=\xi_{3}=$ $\xi=0,1,1010^{2}$ in $d=3$ spatial dimensions. 
constant at its initial value until it starts decreasing as $t^{-1 / 2}$ for times $t \gg \tau_{D}\left(1+2 \xi^{2}\right)$.

[98] Figure 7b shows the behavior of the effective dispersion variance for varying source sizes. It increases from its zero initial value according to $t^{2}$ toward a maximum that is attained at times of the order of $\tau_{D}\left(1+2 \xi^{2}\right)$. Then it decreases to zero as $t^{-1 / 2}$ (see equation (93)). Initially, the uncertainty on effective dispersion is zero because at $t=0$ it is equal to the deterministic local dispersion. As the plume is transported in the spatially fluctuating flow field, the plume heterogeneity increases and thus the uncertainty on effective dispersion. The maximum is reached at the time the solute needs to diffuse over a distance larger than the correlation length and the initial source dimension. This is the time, when the solute has sampled the medium heterogeneity by local dispersion and the plume is expected to homogenize. It is interesting to note that the variance values at $t<\tau_{D}\left(1+2 \xi^{2}\right)$ decrease with increasing source size, while the time at which the maximum is attained is shifted. The plume samples initially more heterogeneity as the source is larger and thus the decrease in uncertainty. As the source is larger, however, the plume needs also more time to lose the memory on the initial source distribution.

[99] Figures 8 and 9 illustrate the behavior of the variances of the absolute and effective dispersion coefficients for an areal source distribution perpendicular to the mean flow direction with $\xi_{1}=0$ and $\xi_{2}=\xi_{3}=\xi=10^{-1}, 1,10,10^{2}$. Figures 8 and 9 are obtained by numerical evaluation of the integral expression (C5). Again, it follows from (86) and $A_{i}^{\gamma}(t)$ defined by (85) that the perturbation theory expressions for the variances of the longitudinal absolute and effective dispersion coefficients are identical. Also, for this case, the expressions for the respective variances in directions transverse to the mean flow direction are equal, $\overline{\delta D_{22}^{\gamma}(t)^{2}}=\overline{\delta D_{33}^{\gamma}(t)^{2}}=\overline{\delta D_{T}^{\gamma}(t)^{2}}$.

[100] Figure 8 shows the behavior of the variance of the longitudinal absolute and effective dispersion coefficient.

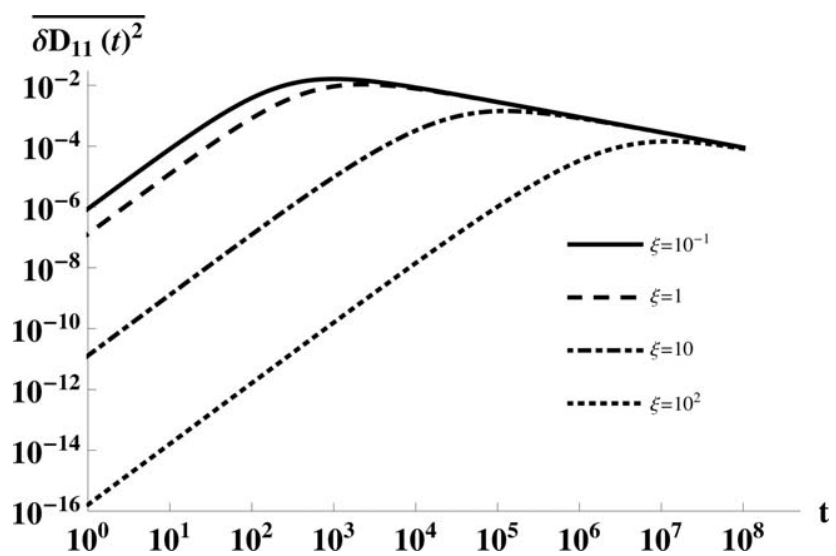

Figure 8. Variance of the longitudinal absolute dispersion coefficient for $\xi_{1}=0$ and the transverse source dimensions $\xi_{1}=\xi_{2}=\xi=10^{-1}, 1,10,10^{2}$ in $d=3$ spatial dimensions. Note that in this case $\overline{\delta D_{11}^{a}(t)^{2}}=$ $\overline{\delta D_{11}^{e}(t)^{2}}=\overline{\delta D_{11}(t)^{2}}$. (a)

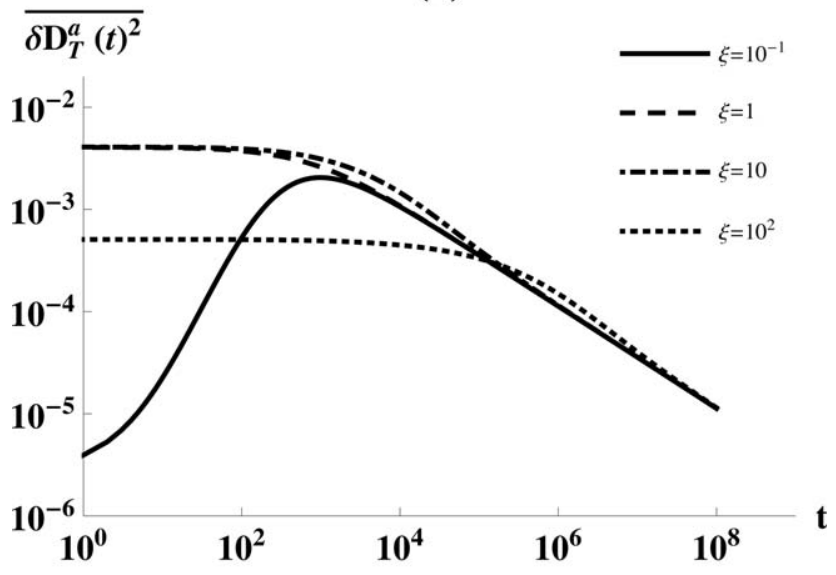

(b)

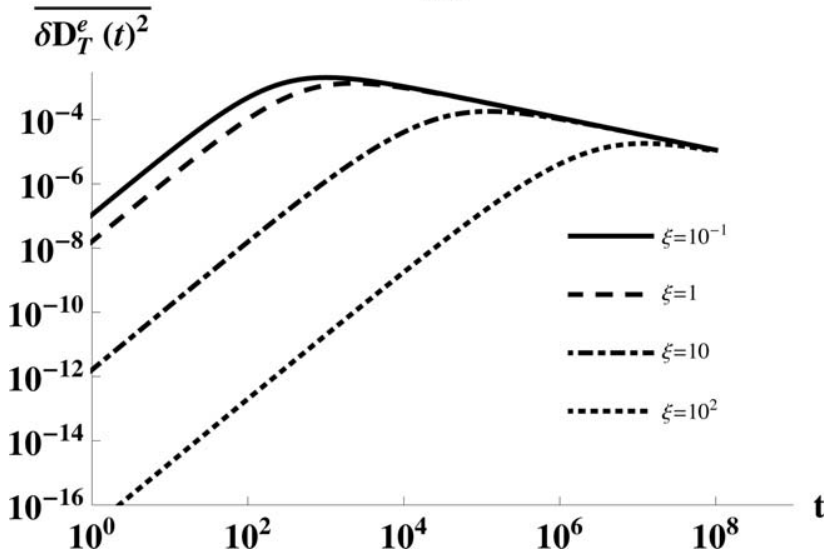

Figure 9. Variance of the transverse (a) absolute and (b) effective dispersion coefficients for $\xi_{1}=0$ and the transverse source dimensions $\xi_{2}=\xi_{3}=\xi=10^{-1}, 1,10,10^{2}$ in $d=3$ spatial dimensions.

The behavior is similar to the one observed for the effective dispersion variance in Figure 7. The preasymptotic variance values decrease with increasing transverse source, while the scale that sets the asymptotic $t^{-1 / 2}$ regime is given by $\tau_{D}\left(1+4 \xi^{2}\right)$.

[101] Similar as in $d=2$, the variances of the transverse coefficients behave differently. Figure 9 shows the transverse dispersion variances for varying areal source dimensions. The absolute transverse dispersion variance, Figure $9 \mathrm{a}$, starts from a nonzero value, which again, reflects the correlation between initial solute injection point and flow velocity. The effective transverse dispersion variance (Figure 9b) shows a similar behavior as for the isotropic source distribution. It evolves as $t^{2}$ toward a maximum that here is attained on the stretched dispersion time scale $\tau_{D}\left(1+4 \xi^{2}\right)$. At asymptotic times, it decreases as $t^{-1 / 2}$.

\section{Summary and Conclusions}

[102] The quantification of sample-to-sample fluctuations is a key question to evaluate the predictive capabilities of stochastic modeling approaches to transport in heterogeneous 
media. Stochastic approaches are oftentimes the only feasible way to systematically quantify the impact of medium heterogeneity on large-scale flow and transport behaviors on one hand and to assess the uncertainty induced by spatial heterogeneity. In this work, we study the self-averaging behavior of solute dispersion in terms of the variance of suitably defined single realization dispersion coefficients about the respective ensemble averages.

[103] We consider three different dispersion concepts for a single medium realization, termed here absolute, effective, and relative dispersion. The absolute dispersion coefficient (20) measures the growth rate of the second centered moment of the solute distribution $c(\mathbf{x}, t)$ in a single realization. Large-scale solute spreading is typically quantified in terms of ensemble averages over the absolute dispersion coefficients. For extended source distributions, its initial value is different from the corresponding local dispersion coefficient (see equation (44)) and related to the correlation between the fluctuations of injection points and the flow velocity within the initial plume. This behavior is insofar counterintuitive as one would expect that an apparent dispersion coefficient at time $t=0$ reflects local-scale dispersion. Furthermore, it implies that the initial variance of the apparent dispersion coefficients may not be zero.

[104] In order to focus on the self-averaging behavior of the actual spreading process in single realizations, we consider two alternative dispersion measures that are defined in terms of the partial plumes that constitute the solute distribution $c(\mathbf{x}, t)$ for finite source distributions. The relative dispersion coefficient (37) quantifies solute spreading with respect to the relative center of mass of the solute distribution; the effective dispersion coefficients (30) are defined as averages of the local effective dispersion coefficients over the source distribution. For a point-like solute injection, the three dispersion quantities coincide.

[105] We show that the ensemble averages of the absolute and relative dispersion coefficients are identical and depend on the source size, while they are different in single realizations. The ensemble average of the effective dispersion coefficient is identical to the one for a point-like injection. Thus, the concept of solute dispersion in single realizations needs to be carefully considered when one wants to quantify dispersion for solute plumes that evolve from extended source distributions.

[106] To study the temporal evolution of variance of the absolute, effective, and relative dispersion coefficients about their respective ensemble averages, we developed explicit expressions for the variance of the center of mass velocity and the respective dispersion coefficients using perturbation theory in the fluctuations of the random flow field, which is assumed to be statistically homogeneous. Therefore, our analytical solutions are strictly valid only for low to mildly heterogeneous stationary random media. Notice also that the choice of the smooth initial condition (3) may underestimate the uncertainty obtained, for example, for a uniform initial distribution or a flux-averaged solute input. The impact of the specific form of the initial distribution on dispersion uncertainty is the subject of future work.

[107] The variances of the effective and relative dispersion coefficients are equal in lowest order perturbation theory, and in general different from the absolute disper- sion variance, which as pointed out earlier, starts from a nonzero initial value. The effective dispersion variance is initially zero because effective dispersion evolves from deterministic local dispersion. Its preasymptotic values decrease as the initial plume size increases. At asymptotically large times, absolute and effective dispersion variances converge. The asymptotic regime is marked by the diffusion time over the characteristic heterogeneity and initial plume sizes.

[108] We find a strong dependence of the self-averaging behavior on the dimensionality of space. In $d=2$ spatial dimensions, the dispersion variances converge to a finite asymptotic values. The dispersion behavior is not selfaveraging, which indicates that the medium heterogeneity cannot be fully sampled by local-scale mass transfer mechanisms. Similar behavior has been found for dispersion in 2-D stratified random flow fields.

[109] For $d=3$ spatial dimensions this is different. Here the dispersion variances tend to zero as $t^{-1 / 2}$ for times much larger than the diffusion scale. Dispersion is selfaveraging. The additional spatial dimension enables for the full sampling of the flow heterogeneity by local mass transfer mechanisms (mixing) and thus for the convergence (in average) of the single realization quantity to its ensemble mean.

[110] Notice that while dispersion coefficients by themselves are rather measures for the plume extension, the evolution of their variances does inform on the mixing efficiency of the transport processes. As the solute becomes better mixed, the dispersion variance decreases because the variability between realization decreases. This is reflected on one hand in the dependence of the variance evolution on the dispersion time scale $\tau_{D}$, and on the other hand, it is reflected in the dependence on dimensionality. Mixing is more efficient in $d=3$ than in $d=2$ dimensions, which leads to the behaviors discussed earlier.

[111] The evolution of the effective dispersion variance contains information on both self-averaging and ergodicity because on one hand, effective dispersion is a single realization processes (self-averaging), and on the other hand, it can be seen as a spatial average over the local effective dispersion coefficients (ergodicity). The averaging volume depends on the source size. As pointed out earlier, we find that the variance decreases at intermediate times as the source size increases, which is in line with the findings of Dagan [1990]. The physical mechanism, however, that leads to a decrease of dispersion variance at asymptotic times is local-scale mass transfer as discussed earlier.

[112] In summary, this work sheds some new light on the concepts of dispersion in single medium realizations and their quantification in terms of ensemble averages in a stochastic modeling framework. The evolution of dispersion variance informs on solute mixing as well as on ergodicity of the transport process. These findings may be relevant for the interpretation of dispersion data from field and laboratory experiments and the assessment of transport uncertainty in the framework of probabilistic risk assessment [e.g., Maxwell et al., 1999; de Barros and Rubin, 2008; Tartakovsky, 2012]. This requires determining the PDF of dispersion coefficients and exploring the impact of dispersion variability on concentration uncertainty. Work in this direction is in progress. 


\section{Appendix A : Mean Center of Mass Velocity}

[113] The center of mass velocity for a plume evolving from a point-like injection at $\mathbf{x}^{\prime}$ can be expressed as

$$
\hat{v}_{i}\left(t \mid \mathbf{x}^{\prime}\right)=\left\langle u_{i}\left[\mathbf{x}\left(t \mid \mathbf{x}^{\prime}\right)\right]\right\rangle=\int \mathrm{d} \mathbf{x} u_{i}(\mathbf{x})\left\langle\delta\left[\mathbf{x}-\mathbf{x}\left(t \mid \mathbf{x}^{\prime}\right)\right]\right\rangle .
$$

[114] For the first equality we used (17) in (27), the second equality is actually an identity. Using now definition (6) for the Green's function in terms of the particle trajectories, we obtain

$$
\hat{v}_{i}\left(t \mid \mathbf{x}^{\prime}\right)=\int \mathrm{d} \mathbf{x} u_{i}(\mathbf{x}) g\left(\mathbf{x}, t \mid \mathbf{x}^{\prime}\right) .
$$

[115] The ensemble average is given by

$$
\overline{\hat{v}_{i}\left(t \mid \mathbf{x}^{\prime}\right)}=\int \mathrm{d} \mathbf{x} \overline{u_{i}(\mathbf{x}) g\left(\mathbf{x}, t \mid \mathbf{x}^{\prime}\right)} .
$$

[116] Using the stationarity of the underlying random field, the integrand can be written as

$$
\overline{\hat{v}_{i}\left(t \mid \mathbf{x}^{\prime}\right)}=\int \mathrm{d} \mathbf{x} \overline{u_{i}\left(\mathbf{x}^{\prime}\right) g\left(\mathbf{x}^{\prime}, t \mid \mathbf{x}\right)} .
$$

[117] The Green's function is normalized with respect to integration over its first and second arguments, respectively,

$$
\int \mathrm{d} \mathbf{x} g\left(\mathbf{x}, t \mid \mathbf{x}^{\prime}\right)=\int \mathrm{d} \mathbf{x}^{\prime} g\left(\mathbf{x}, t \mid \mathbf{x}^{\prime}\right)=1
$$

as a consequence of the incompressibility of the random velocity field $\mathbf{u}(\mathbf{x})$. Thus, we obtain for the mean center of mass velocity

$$
\overline{\hat{v}_{i}\left(t \mid \mathbf{x}^{\prime}\right)}=\overline{u_{i}\left(\mathbf{x}^{\prime}\right)}=\delta_{i 1} \bar{u} .
$$

[118] This can also be seen explicitly by using perturbation theory. To this effect, we insert $u_{i}(\mathbf{x})=\bar{u} \delta_{i 1}+u_{i}^{\prime}(\mathbf{x})$ into (A2) and write it in terms of the Fourier transform of the Green's function $g\left(\mathbf{x}, t \mid \mathbf{x}^{\prime}\right)$ as

$$
\hat{v}_{i}\left(t \mid \mathbf{x}^{\prime}\right)=\bar{u} \delta_{i 1}+\int_{k} \tilde{u}_{i}^{\prime}(\mathbf{k}) \tilde{g}\left(-\mathbf{k}, t \mid \mathbf{x}^{\prime}\right) .
$$

[119] The perturbation expansion for $\tilde{g}\left(-\mathbf{k}, t \mid \mathbf{x}^{\prime}\right)$ is obtained by using the initial condition $\tilde{\rho}(\mathbf{k})=\exp \left(i \mathbf{k} \cdot \mathbf{x}^{\prime}\right)$ in (59) and (60)-(62). Inserting this perturbation expansion up to second order in the velocity field fluctuations into (A7) gives for the second term on the right side of (A7)

$$
\begin{aligned}
& \int_{k} \tilde{u}_{i}^{\prime}(\mathbf{k}) \tilde{g}\left(-\mathbf{k}, t \mid \mathbf{x}^{\prime}\right)=\int_{k} \tilde{u}_{i}^{\prime}(\mathbf{k}) \tilde{g}_{0}\left(-\mathbf{k}, t \mid \mathbf{x}^{\prime}\right) \\
& \quad+\int_{k} \tilde{u}_{i}^{\prime}(\mathbf{k}) \int_{0}^{t} d t^{\prime} \tilde{g}_{0}\left(\mathbf{k}, t-t^{\prime}\right) \int_{k^{\prime}} i \mathbf{k} \cdot \tilde{\mathbf{u}}^{\prime}\left(\mathbf{k}^{\prime}\right) \tilde{g}_{0}\left(\mathbf{k}-\mathbf{k}^{\prime}, t^{\prime} \mid \mathbf{x}^{\prime}\right) \\
& \quad+\int_{k}^{t} \tilde{u}_{i}^{\prime}(\mathbf{k}) \int_{0}^{t} d t^{\prime} \tilde{g}_{0}\left(\mathbf{k}, t-t^{\prime}\right) \int_{k^{\prime}} i \mathbf{k} \cdot \tilde{\mathbf{u}}^{\prime}\left(\mathbf{k}^{\prime}\right) \int_{0}^{t^{\prime}} d \mathbf{t}^{\prime \prime} \tilde{g}_{0}\left(\mathbf{k}-\mathbf{k}^{\prime}, t^{\prime}-\mathbf{t}^{\prime \prime}\right) \\
& \quad \times \int_{k^{\prime \prime}} i\left(\mathbf{k}-\mathbf{k}^{\prime}\right) \cdot \tilde{\mathbf{u}}^{\prime}\left(\mathbf{k}^{\prime \prime}\right) \tilde{g}_{0}\left(\mathbf{k}-\mathbf{k}^{\prime}-\mathbf{k}^{\prime \prime}, \mathbf{t}^{\prime \prime} \mid \mathbf{x}^{\prime}\right),
\end{aligned}
$$

where we defined $\tilde{g}_{0}\left(\mathbf{k}, t \mid \mathbf{x}^{\prime}\right)=\tilde{g}_{0}(\mathbf{k}, t) \exp \left(i \mathbf{k} \cdot \mathbf{x}^{\prime}\right)$. Performing the ensemble average on both sides of (A8) one obtains that

$$
\int_{k} \overline{\tilde{u}_{i}^{\prime}(\mathbf{k}) \tilde{g}\left(-\mathbf{k}, t \mid \mathbf{x}^{\prime}\right)}=0 .
$$

[120] The first term on the right side of (A8) is zero because $\overline{\tilde{u}_{i}(\mathbf{k})}=0$. The second term on the right side of (A8) is zero because

$$
\overline{\tilde{u}_{i}^{\prime}(\mathbf{k}) \mathbf{k} \cdot \tilde{\mathbf{u}}^{\prime}\left(\mathbf{k}^{\prime}\right)}=k_{j} \tilde{C}_{i j}(\mathbf{k}) \delta\left(\mathbf{k}+\mathbf{k}^{\prime}\right)=0,
$$

where we use the Einstein convention of summation over equal indices. The first equal sign on the right side of (A10) is a consequence of the fact that the velocity covariance is translation-invariant, and the second equal sign results from the fact that the flow field is divergence-free. Thus, we confirm explicitly in second-order perturbation theory that the mean center of mass velocity is equal to the Eulerian mean flow velocity.

\section{Appendix B : Variance of Center of Mass Velocity}

[121] We obtain for the variance of the center of mass velocity explicit closed form expressions in $d=2$ and compact integral expression in $d=3$ as outlined in the following.

\section{B1. Two Spatial Dimensions}

[122] We obtain by evaluating (80) for the fully anisotropic case in $d=2$

$$
\begin{gathered}
\delta v_{1}^{2}(t)=\frac{\sigma^{2} \bar{u}^{2} l_{1}\left[2 B_{1}(t) \eta+B_{2}(t)\right]}{2 l_{2} B_{2}(t)\left[B_{1}(t) \eta+B_{2}(t)\right]^{2}} . \\
\delta v_{2}^{2}(t)=\frac{\sigma^{2} \bar{u}^{2} l_{1}}{2 l_{2}\left[B_{1}(t) \eta+B_{2}(t)\right]^{2}},
\end{gathered}
$$

where we defined $\eta=l_{1} / l_{2}$.

\section{B2. Three Spatial Dimensions}

[123] For the fully anisotropic case, we obtain for $d=3$ from (80) the compact integral expressions

$$
\delta v_{i}^{2}(t)=3 \sigma^{2} \bar{u}^{2} \int_{0}^{\infty} d \lambda h_{i}(\lambda, t)
$$

[124] The auxiliary functions $h_{i}(\lambda, t)$ in (B3) are given by

$$
\begin{aligned}
h_{1}(\lambda, t)= & \frac{3 \sigma^{2} \bar{u}^{2} \lambda}{\left[2 \lambda+B_{1}(t)^{2} l_{1}^{2}\right]^{1 / 2}\left[2 \lambda+B_{2}(t)^{2} l_{2}^{2}\right]^{1 / 2}\left[2 \lambda+B_{3}(t)^{2} l_{3}^{2}\right]^{5 / 2}} \\
& +\frac{3 \sigma^{2} \bar{u}^{2} \lambda}{\left[2 \lambda+B_{1}(t)^{2} l_{1}^{2}\right]^{1 / 2}\left[2 \lambda+B_{2}(t)^{2} l_{2}\right]^{5 / 2}\left[2 \lambda+B_{3}(t)^{2} l_{3}^{2}\right]^{1 / 2}} \\
& +\frac{2 \sigma^{2} \bar{u}^{2} \lambda}{\left[2 \lambda+B_{1}(t)^{2} l_{1}^{2}\right]^{1 / 2}\left[2 \lambda+B_{2}(t)^{2} l_{2}^{2}\right]^{3 / 2}\left[2 \lambda+B_{3}(t)^{2} l_{3}^{2}\right]^{3 / 2}}
\end{aligned}
$$


DENTZ AND DE BARROS: DISPERSION VARIANCE IN HETEROGENEOUS MEDIA

$$
\begin{aligned}
& h_{2}(\lambda, t)=\frac{\sigma^{2} \bar{u}^{2} \lambda}{\left[2 \lambda+B_{1}(t)^{2} l_{1}^{2}\right]^{3 / 2}\left[2 \lambda+B_{2}(t)^{2} l_{2}^{2}\right]^{3 / 2}\left[2 \lambda+B_{3}(t)^{2} l_{3}^{2}\right]^{1 / 2}} \\
& h_{3}(\lambda, t)=\frac{\sigma^{2} \bar{u}^{2} \lambda}{\left[2 \lambda+B_{1}(t)^{2} l_{1}^{2}\right]^{3 / 2}\left[2 \lambda+B_{2}(t)^{2} l_{2}^{2}\right]^{1 / 2}\left[2 \lambda+B_{3}(t)^{2} l_{3}^{2}\right]^{3 / 2}}
\end{aligned}
$$

\section{Appendix C: Variance of Dispersion Coefficients}

[125] We obtain for the variance of the dispersion coefficients explicit closed form expressions in $d=2$ and compact integral expression in $d=3$ as outlined in the following.

[126] Inserting (55) for $\tilde{g}_{0}(\mathbf{k}, t),(56)$ for $\tilde{\rho}(\mathbf{k})$, and (10) for $\tilde{C}(\mathbf{k})$ into (86), we obtain

$$
\begin{aligned}
\overline{\delta D_{i j}^{\gamma}(t)^{2}}= & \sigma^{2} \bar{u}^{2} \frac{(2 \pi)^{d / 2}}{4} \int_{k} \prod_{n=1}^{d} l_{n} \exp \left[\frac{k_{n}^{2} l_{n}^{2} B_{n}(t)^{2}}{2}\right] \\
& \times\left[p_{i}(\mathbf{k})^{2} k_{j}^{2} A_{j}^{\gamma}(t)^{4} l_{j}^{4}+p_{j}(\mathbf{k})^{2} k_{i}^{2} A_{i}^{\gamma}(t)^{4} l_{i}^{4}\right. \\
& \left.+2 p_{i}(\mathbf{k}) p_{j}(\mathbf{k}) k_{i} k_{j} A_{i}^{\gamma}(t)^{2} A_{j}^{\gamma}(t)^{2} l_{i}^{2} l_{j}^{2}\right]
\end{aligned}
$$

where the $B_{i}(t)$ are defined by $(81)$.

\section{C1. Two Spatial Dimensions}

[127] We obtain by evaluating (C1) for the fully anisotropic case in $d=2$

$$
\begin{gathered}
\delta D_{11}^{\gamma}(t)^{2}=\sigma^{2} \bar{u}^{2} l_{1}^{2}\left[\frac{A_{1}^{\gamma}(t)}{B_{2}(t)}\right]^{4}\left[\frac{B_{1}(t)}{B_{2}(t)}+\eta^{-1}\right]^{-3} \\
\delta D_{22}^{\gamma}(t)^{2}=\sigma^{2} \bar{u}^{2} l_{2}^{2} \eta^{-2}\left[\frac{A_{2}^{\gamma}(t)}{B_{2}(t)}\right]^{4}\left[\frac{B_{1}(t)}{B_{2}(t)}+\eta^{-1}\right]^{-3} \\
\delta D_{12}^{\gamma}(t)^{2}=\frac{1}{4} \sigma^{2} \bar{u}^{2} l_{2}^{2} \eta^{-2}\left[\frac{A_{2}^{\gamma}(t)}{B_{2}(t)}\right]^{4} \frac{B_{1}(t)^{2} \eta^{2}+3 B_{1}(t) B_{2}(t) \eta+B_{2}(t)^{2}}{B_{2}(t)^{2}} \\
\left.+\frac{B_{1}(t)}{B_{2}(t)}+\eta^{-1}\right]^{-3} \\
+\frac{1}{4} \sigma^{2} \bar{u}^{2} l_{1} l_{2} \eta^{-2} \frac{A_{1}^{\gamma}(t)^{4}}{B_{1}(t) B_{2}(t)^{3}}\left[\frac{B_{1}(t)}{B_{2}(t)}+\eta^{-1}\right]^{-3} \\
-\frac{1}{2} \sigma^{2} \bar{u}^{2} l_{2}^{2} \frac{A_{1}^{\gamma}(t)^{2} A_{2}^{\gamma}(t)^{2}}{B_{2}(t)^{4}}\left[\frac{B_{1}(t)}{B_{2}(t)}+\eta^{-1}\right]^{-3}
\end{gathered}
$$

where $\eta=l_{1} / l_{2}$.

\section{C2. Three Spatial Dimensions}

[128] For the fully anisotropic case, we obtain for $d=3$ from $(\mathrm{C} 1)$ the compact integral expressions

$$
\delta D_{i j}^{\gamma}(t)^{2}=\sigma^{2} \bar{u}^{2} l_{i} l_{j} \int_{0}^{\infty} d \lambda \lambda f_{i j}^{\gamma}(\lambda, t)
$$

where the auxiliary functions $f_{i j}^{\gamma}(\lambda, t)$ are given by

$$
\begin{aligned}
f_{11}^{\gamma}(\lambda, t)= & l_{1}^{3} l_{2} l_{3} A_{1}^{\gamma}(t)^{4} \frac{3 B_{3}(t)^{4} l_{3}^{4}+16 B_{3}(t)^{2} l_{3}^{3} \lambda+32 \lambda^{2}+2 B_{2}(t)^{2} l_{2}^{2} B_{3}(t)^{2} l_{3}^{2}+16 B_{2}(t)^{2} l_{2}^{2} \lambda+3 B_{2}(t)^{4} l_{2}^{4}}{\left[B_{1}(t)^{2} l_{1}^{2}+2 \lambda\right]^{3 / 2}\left[B_{2}(t)^{2} l_{2}^{2}+2 \lambda\right]^{5 / 2}\left[B_{3}(t)^{2} l_{3}^{2}+2 \lambda\right]^{5 / 2}} \\
& f_{22}^{\gamma}(\lambda, t)=l_{1} l_{2}^{3} l_{3} A_{2}^{\gamma}(t)^{4} \frac{1}{\left[B_{1}(t)^{2} l_{1}^{2}+2 \lambda\right]^{3 / 2}\left[B_{2}(t)^{2} l_{2}^{2}+2 \lambda\right]^{5 / 2}\left[B_{3}(t)^{2} l_{3}^{2}+2 \lambda\right]^{1 / 2}} \\
& f_{33}^{\gamma}(\lambda, t)=l_{1} l_{2} l_{3}^{3} A_{3}^{\gamma}(t)^{4} \frac{1}{\left[B_{1}(t)^{2} l_{1}^{2}+2 \lambda\right]^{3 / 2}\left[B_{2}(t)^{2} l_{2}^{2}+2 \lambda\right]^{1 / 2}\left[B_{3}(t)^{2} l_{3}^{2}+2 \lambda\right]^{5 / 2}} \\
f_{12}^{\gamma}(\lambda, t)= & \frac{3}{4} l_{2}^{4} l_{3} A_{2}^{\gamma}(t)^{4} \frac{5 B_{3}(t)^{4} l_{3}^{4}+24 B_{3}(t)^{2} l_{3}^{2} \lambda+32 \lambda^{2}+2 B_{2}(t)^{2} l_{2}^{2} B_{3}(t)^{2} l_{3}^{2}+8 B_{2}(t)^{2} l_{2}^{2} \lambda+B_{2}(t)^{4} l_{2}^{4}}{\left[B_{1}(t)^{2} l_{1}^{2}+2 \lambda\right]^{1 / 2}\left[B_{2}(t)^{2} l_{2}^{2}+2 \lambda\right]^{7 / 2}\left[B_{3}(t)^{2} l_{3}^{2}+2 \lambda\right]^{5 / 2}} \\
& +\frac{3}{4} l_{1}^{4} l_{3} A_{1}^{\gamma}(t)^{4} \frac{1}{\left[B_{1}(t)^{2} l_{1}^{2}+2 \lambda\right]^{5 / 2}\left[B_{2}(t)^{2} l_{2}^{2}+2 \lambda\right]^{3 / 2}\left[B_{3}(t)^{2} l_{3}^{2}+2 \lambda\right]^{1 / 2}} \\
& -2 l_{1}^{2} l_{2}^{2} l_{3} A_{1}^{\gamma}(t)^{2} A_{2}^{\gamma}(t)^{2} \frac{3 B_{3}(t)^{2} l_{3}^{2}+8 \lambda+B_{2}(t)^{2}}{\left[B_{1}(t)^{2} l_{1}^{2}+2 \lambda\right]^{3 / 2}\left[B_{2}(t)^{2} l_{2}^{2}+2 \lambda\right]^{5 / 2}\left[B_{3}(t)^{2} l_{3}^{2}+2 \lambda\right]^{3 / 2}} \\
& \\
& \\
&
\end{aligned}
$$


DENTZ AND DE BARROS: DISPERSION VARIANCE IN HETEROGENEOUS MEDIA

$$
\begin{aligned}
f_{13}^{\gamma}(\lambda, t)= & \frac{3}{4} l_{3}^{4} l_{2} A_{3}^{\gamma}(t)^{4} \frac{5 B_{2}(t)^{4} l_{2}^{4}+24 B_{2}(t)^{2} l_{2}^{2} \lambda+32 \lambda^{2}+2 B_{2}(t)^{2} l_{2}^{2} B_{3}(t)^{2} l_{3}^{2}+8 B_{3}(t)^{2} l_{3}^{2} \lambda+B_{3}(t)^{4} l_{3}^{4}}{\left[B_{1}(t)^{2} l_{1}^{2}+2 \lambda\right]^{1 / 2}\left[B_{2}(t)^{2} l_{2}^{2}+2 \lambda\right]^{5 / 2}\left[B_{3}(t)^{2} l_{3}^{2}+2 \lambda\right]^{7 / 2}} \\
& +\frac{3}{4} l_{1}^{4} l_{2} A_{1}^{\gamma}(t)^{4} \frac{1}{\left[B_{1}(t)^{2} l_{1}^{2}+2 \lambda\right]^{5 / 2}\left[B_{2}(t)^{2} l_{2}^{2}+2 \lambda\right]^{1 / 2}\left[B_{3}(t)^{2} l_{3}^{2}+2 \lambda\right]^{3 / 2}} \\
& -2 l_{1}^{2} l_{3}^{2} l_{2} A_{1}^{\gamma}(t)^{2} A_{3}^{\gamma}(t)^{2} \frac{3 B_{2}(t)^{2} l_{3}^{2}+8 \lambda+B_{3}(t)^{2}}{\left[B_{1}(t)^{2} l_{1}^{2}+2 \lambda\right]^{3 / 2}\left[B_{2}(t)^{2} l_{2}^{2}+2 \lambda\right]^{3 / 2}\left[B_{3}(t)^{2} l_{3}^{2}+2 \lambda\right]^{5 / 2}}
\end{aligned}
$$

$$
\begin{aligned}
& f_{23}^{\gamma}(\lambda, t) \\
& =\frac{l_{1}}{4} \frac{A_{3}^{\gamma}(t)^{4} l_{3}^{4}+A_{2}^{\gamma}(t)^{4} l_{2}^{4}+2 A_{2}^{\gamma}(t)^{2} l_{2}^{2} A_{3}^{\gamma}(t)^{2} l_{3}^{2}}{\left[B_{1}(t)^{2} l_{1}^{2}+2 \lambda\right]^{3 / 2}\left[B_{2}(t)^{2} l_{2}^{2}+2 \lambda\right]^{3 / 2}\left[B_{3}(t)^{2} l_{3}^{2}+2 \lambda\right]^{3 / 2}}
\end{aligned}
$$

\section{C3. Asymptotic Behavior}

[129] We consider here the asymptotic long time behavior for the variance of the diagonal dispersion coefficient, $i=j$. Rescaling $k_{i}^{\prime}=k_{i} l_{i} B_{i}(t)$ in $(\mathrm{C} 1)$, we obtain

$$
\begin{aligned}
\overline{\delta D_{i i}^{\gamma}(t)^{2}}= & \sigma^{2} \bar{u}^{2} l_{i}^{2}(2 \pi)^{d / 2} \frac{A_{i}^{\gamma}(t)^{4}}{B_{i}(t)^{2}} \prod_{n=1}^{d} B_{n}(t)^{-1} \times \int_{k^{\prime}} \exp \left(\frac{k^{\prime 2}}{2}\right) k_{i}^{\prime 2} \\
& {\left[\delta_{i 1}-\frac{k_{1}^{\prime} k_{i}^{\prime}}{\sum_{n=1}^{d} k_{n}^{\prime 2} l_{1} l_{i} / l_{n}^{2} B_{1}(t) B_{i}(t) / B_{n}(t)^{2}}\right] . }
\end{aligned}
$$

[130] Note that in the limit $t \rightarrow \infty$, we obtain

$$
\lim _{t \rightarrow \infty} \frac{B_{1}(t) B_{i}(t)}{B_{n}(t)^{2}}=\frac{\tau_{D_{n}}}{\sqrt{\tau_{D_{1}} \tau_{D_{i}}}} .
$$

[131] For $d=2$, we obtain in this limit for the first term on the right side of $(\mathrm{C} 12)$

$$
\lim _{t \rightarrow \infty} \frac{A_{i}^{\gamma}(t)^{4}}{B_{i}(t)^{2} B_{1}(t) B_{2}(t)}=\frac{1}{4} \frac{\sqrt{\tau_{D_{1}} \tau_{D_{2}}}}{\tau_{D_{i}}}
$$

and in $d=3$

$$
\lim _{t \rightarrow \infty} \frac{A_{i}^{\gamma}(t)^{4}}{B_{i}(t)^{2} B_{1}(t) B_{2}(t) B_{3}(t)}=\frac{1}{8} \frac{\sqrt{\tau_{D_{1}} \tau_{D_{2}} \tau_{D_{3}}}}{\tau_{D_{i}}} t^{-1 / 2} .
$$

[132] Thus, we obtain for the asymptotic behavior of the dispersion variance in $d=2$ in the limit of large times

$$
\begin{aligned}
\overline{\delta D_{i j}^{\gamma}(t)^{2}=} & \sigma^{2} \bar{u}^{2} l_{1} l_{2}(2 \pi) \frac{D_{i}}{4 \sqrt{D_{1} D_{2}}} \\
& \int_{k^{\prime}} \exp \left(\frac{k^{\prime} 2}{2}\right) k_{i}^{\prime 2}\left(\delta_{i 1}-\frac{k_{1}{ }^{\prime} k_{i}{ }^{\prime}}{\sum_{n=1}^{2} k_{n}^{\prime 2} \sqrt{D_{1} D_{i}} / D_{n}}\right) .
\end{aligned}
$$

[133] Thus, in $d=2$ dimensions, the variance tends toward a constant, and the dispersion coefficients are not self-averaging quantities. For $d=3$ dimensions, this is different. We find

$$
\begin{aligned}
\overline{\delta D_{i i}^{\gamma}(t)^{2}}= & \sigma^{2} \bar{u}^{2}(2 \pi)^{3 / 2} l_{1} l_{2} l_{3} \frac{D_{i}}{8 \sqrt{D_{1} D_{2} D_{3}}} t^{-1 / 2} \\
& \int_{k^{\prime}} \exp \left(\frac{k^{\prime} 2}{2}\right) k_{i}^{\prime 2}\left(\delta_{i 1}-\frac{k_{1}{ }^{\prime} k_{i}^{\prime}}{\sum_{n=1}^{3} k_{n}^{\prime 2} \sqrt{D_{1} D_{i}} / D_{n}}\right) .
\end{aligned}
$$

[134] Thus, the dispersion coefficients are self-averaging quantities in $d=3$. Furthermore, we see that in the case of isotropic dispersion, $D_{i}=D$ for $i=1, \cdots d$, the asymptotic behavior in $d=2$ dimensions is given by

$$
\overline{\delta D_{i i}^{\gamma}(t)^{2}}=\frac{1}{32} \sigma^{2} \bar{u}^{2} l_{1} l_{2}
$$

[135] The asymptotic long time value is the same for all $i$. For $d=3$ spatial dimensions, we obtain

$$
\overline{\delta D_{i i}^{\gamma}(t)^{2}}=l_{1} l_{2} l_{3} \frac{t^{-1 / 2}}{4 \sqrt{D}} a_{i}
$$

with $a_{1}=1 / 35$ and $a_{2}=a_{3}=3 / 280$.

\section{Notation}

$\begin{aligned} & A_{n}^{a}, A_{n}^{e} \text { auxiliary function defined in }(85) . \\ & a_{i} \text { coefficients. } \\ & B_{n} \text { function defined in }(81) . \\ & c \text { concentration field. } \\ & \tilde{c_{o}}, \tilde{c_{1}}, \tilde{c_{2}} \quad \text { zeroth-, first-, and second-order concentra- } \\ & \quad \text { tion contributions in Fourier space. } \\ & C \quad \text { log-conductivity covariance function. } \\ & C_{i j} \text { velocity covariance function. } \\ & d \text { flow dimensionality. } \\ & \mathbf{D} \text { local-scale dispersion tensor. } \\ & D_{i j}^{e}, D_{i j}^{r} \text { absolute, effective, and relative dispersion } \\ & \text { coefficients. } \\ & f \text { log-conductivity. } \\ & f^{\prime} \text { fluctuations of the log-conductivity. } \\ & h \text { hydraulic head. } \\ & g \text { the Green's function. } \\ & G \text { hydraulic gradient. } \\ & i \text { imaginary unit. } \\ & i, n, j \text { indices. } \\ & K_{g} \text { geometric mean } \\ & \text { conductivity. }\end{aligned}$




\author{
k wave number vector. \\ $l_{i} \quad$ correlation length in the $i$ th direction. \\ $L_{i}$ dimension of the source in the $i$ th direction. \\ $m_{i}^{a}, m_{i j}^{a} \quad$ absolute spatial moments. \\ $m_{i}^{r}, m_{i j}^{r} \quad$ relative spatial moments. \\ $\hat{m}_{i}, \hat{m}_{i j} \quad$ local spatial moments for a point source. \\ Pe Péclet number. \\ $t$ time. \\ u velocity vector. \\ $\bar{u}$ mean velocity along the longitudinal \\ direction. \\ $v_{i}^{a}, v_{i}^{r} \quad$ absolute and relative center of mass velocity. \\ $\mathbf{x}$ coordinate vector.
}

\section{Greek Symbols}

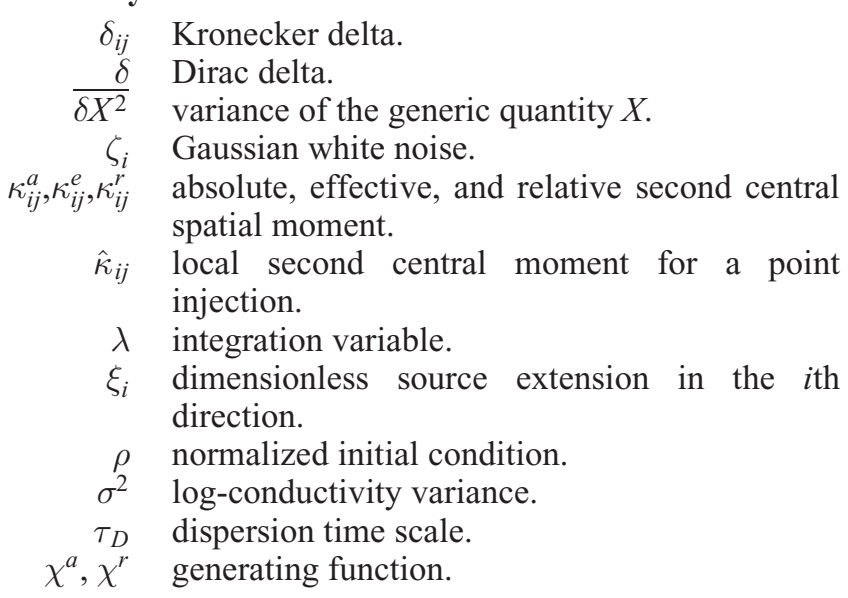

\section{Special Symbols}

$\bar{X}$ expected value of the generic quantity $X$.

$\tilde{X}$ Fourier transform of the generic quantity $X$.

[136] Acknowledgments. F.P.J.B. acknowledges the partial support of the Spanish Juan de la Cierva program. MD acknowledges the support of the FP7 EU project PANACEA (grant agreement 282900) and the Spanish Ministry of Economy and Competitivity through the project HEART (CGL2010-18450). The authors would like to thank Alberto Bellin, Reed Maxwell, and two anonymous reviewers for the valuable comments which helped to improve the clarity of this manuscript.

\section{References}

Attinger, S., M. Dentz, H. Kinzelbach, and W. Kinzelbach (1999), Temporal behaviour of a solute cloud in a chemically heterogeneous porous medium, J. Fluid Mech., 386, 77-104.

Bear, J. (1972), Dynamics of Fluids in Porous Media, Elsevier Sci., New York.

Bellin, A., M. Pannone, A. Fiori, and A. Rinaldo (1996), On transport in porous formations characterized by heterogeneity of evolving scales, Water Resour. Res., 32, 3485-3496.

Berkowitz, B., A. Cortis, M. Dentz, and H. Scher (2006), Modeling nonFickian transport in geological formations as a continuous time random walk, Rev. Geophys., 44, RG2003, doi:10.1029/2005RG000178.

Boggs, J. M., S. C. Young, L. M. Beard, L. W. Gelhar, K. R. Rehfeldt, and E. E. Adams (1992), Field study of dispersion in a heterogeneous aquifer: 1. Overview and site description, Water Resour. Res., 28, 32813291 .

Bouchaud, J. P., and A. Georges (1990), Anomalous diffusion in disordered media: Statistical mechanisms, models and physical applications, Phys. Rep., 195(4-5), 127-293.

Cirpka, O., F. de Barros, G. Chiogna, and W. Nowak (2011a), Probability density function of steady state concentration in two-dimensional heterogeneous porous media, Water Resour. Res., 47, W11523, doi:10.1029/ 2011WR010750.
Cirpka, O., F. P. J. de Barros, G. Chiogna, M. Rolle, and W. Nowak (2011b), Stochastic flux-related analysis of transverse mixing in twodimensional heterogeneous porous media, Water Resour. Res., 47, W07540, doi:10.1029/2010WR010279.

Clincy, M., and H. Kinzelbach (2001), Stratified disordered media: Exact solutions for transport parameters and their self-averaging properties, J. Phys. A: Math. Gen., 34, 7141-7152.

Cushman, J. H., X. Hu, and T. R. Ginn (1994), Nonequilibrium statistical mechanics of preasymptotic dispersion, J. Stat. Phys., 75(5/6), 859-878.

Dagan, G. (1984), Solute transport in heterogeneous porous formations, J. Fluid Mech., 145, 151-177.

Dagan, G. (1988), Time-dependent macrodispersion for solute transport in anisotropic heterogeneous aquifers, Water Resour. Res., 24(9), 1491-1500.

Dagan, G. (1990), Transport in heterogeneous porous formations: Spatial moments, ergodicity, and effective dispersion, Water Resour. Res., 26(6), 1281-1290.

Dagan, G. (1991), Dispersion of a passive solute in non-ergodic transport by steady velocity fields in heterogeneous formations, J. Fluid Mech., 233, 197-210.

de Barros, F. P. J., and Y. Rubin (2008), A risk-driven approach for subsurface site characterization, Water Resour. Res., 44, W01414, doi:10.1029/ 2007WR006081.

de Barros, F. P. J., and Y. Rubin (2011), Modelling of block-scale macrodispersion as a random function, J. Fluid Mech., 676(1), 514-545.

Dentz, M., and J. Carrera (2007), Mixing and spreading in stratified flow, Phys. Fluids, 19, 017,107.

Dentz, M., and D. Tartakovsky (2010), Probability density functions for passive scalars dispersed in random velocity fields, Geophys. Res. Lett., 37, L24406, doi:10.1029/2010GL045748.

Dentz, M., H. Kinzelbach, S. Attinger, and W. Kinzelbach (2000a), Temporal behaviour of a solute cloud in a heterogeneous porous medium: 1. Point-like injection, Water Resour. Res., 36, 3591-3604.

Dentz, M., H. Kinzelbach, S. Attinger, and W. Kinzelbach (2000b), Temporal behaviour of a solute cloud in a heterogeneous porous medium: 2. Spatially extended injection, Water Resour. Res., 36(12), 3605-3614.

Dentz, M., T. Le Borgne, A. Englert, and B. Bijeljic (2010), Mixing, spreading and reaction in heterogeneous media: A brief review, J. Contam. Hydrol., 120-121, 1-17.

Eberhard, J. (2004), Approximations for transport parameters and selfaveraging properties for point-like injections in heterogeneous media, J. Phys. A: Math. Gen. A, 37, 2549-2571.

Eberhard, J., N. Suciu, and C. Vamos (2007), On the self-averaging of dispersion for transport in quasi-periodic random media, J. Phys. A: Math. Theor., 40, 597-610.

Fiori, A. (2001), The Lagrangian concentration approach for determining dilution in aquifer transport: Theoretical analysis and comparison with field experiments, Water Resour. Res., 37, 3105-3114.

Fiori, A., and G. Dagan (2000), Concentration fluctuations in aquifer transport: A rigorous first-order solution and applications, J. Contam. Hydrol., 45, 139-163.

Fiori, A., and I. Janković (2005), Can we determine the transverse macrodispersivity by using the method of moments?, Adv. Water Resour., 28(6), 589-599.

Gelhar, L. W., and C. L. Axness (1983), Three-dimensional stochastic analysis of macrodispersion in aquifers, Water Resour. Res., 19(1), 161-180.

Gelhar, L. W., C. Welty, and K. R. Rehfeldt (1992), A critical review of data on field-scale dispersion in aquifers, Water Resour. Res., 28(7), 1955-1974.

Graham, W., and D. McLaughlin (1989), Stochastic analysis of nonstationary subsurface solute transport: 1. Unconditional moments, Water Resour. Res., 25, 215-232.

Haggerty, R., S. A. McKenna, and L. C. Meigs (2000), On the late time behavior of tracer test breakthrough curves, Water Resour. Res., 36(12), 3467-3479.

Kitanidis, P. K. (1988), Predictions by the method of moments of transport in heterogeneous formations, J. Hydrol., 102(1-4), 453-473.

Maxwell, R., W. E. Kastenberg, and Y. Rubin (1999), A methodology to integrate site characterization information into groundwater-driven health risk assessment, Water Resour. Res., 35(9), 2841-2885.

Neuman, S. P. (1993), Eulerian-Lagrangian theory of transport in space-time nonstationary velocity fields: Exact nonlocal formalism by conditional moments and weak approximation, Water Resour. Res., 29(3), 633-645.

Neuman, S. P., and D. M. Tartakovsky (2008), Perspective on theories of anomalous transport in heterogeneous media, Adv. Water Resour., 32, 670-680, doi:10.1016/j.advwatres.2008.08.005. 


\section{DENTZ AND DE BARROS: DISPERSION VARIANCE IN HETEROGENEOUS MEDIA}

Neuman, S. P., C. L. Winter, and C. M. Newman (1987), Stochastic theory of field-scale Fickian dispersion in anisotropic porous media, Water Resour. Res., 23(3), 453-466.

Rajaram, H., and L. W. Gelhar (1993), Plume scale-dependent dispersion in heterogeneous aquifers: 2 . Eulerian analysis and three-dimensional aquifers, Water Resour. Res., 29(9), 3261-3276.

Rubin, Y. (2003), Applied Stochastic Hydrogeology, Oxford Univ. Press, Oxford.

Rubin, Y., A. Sun, R. Maxwell, and A. Bellin (1999), The concept of blockeffective macrodispersivity and a unified approach for grid-scale- and plume-scale-dependent transport, J. Fluid Mech., 395, 161-180.

Sposito, G., and G. Dagan (1994), Predicting solute plume evolution in heterogeneous porous formations, Water Resour. Res., 30(2), 585-589.

Suciu, N., C. Vamos, J. Vanderborght, H. Hardelauf, and H. Vereecken (2006), Numerical investigations on ergodicity of solute transport in heterogeneous aquifers, Water Resour. Res., 42, W04409, doi:10.1029 2005 WR004546.
Suciu, N., C. Vamos, H. Vereecken, K. Sabelfeld, and P. Knabner (2008), Memory effects induced by dependence on initial conditions and ergodicity of transport in heterogeneous media, Water Resour. Res., 44, W08501, doi:10.1029/2007WR006740.

Suciu, N., C. Vamos, F. A. Radu, H. Vereecken, and P. Knabner (2009), Persistent memory of diffusing particles, Phys. Rev. E, 80, 061134.

Tartakovsky, D. M. (2012), Assessment and management of risk in subsurface hydrology: A review and perspective, Adv. Water Resour., 51 , doi:10.1016/j.advwatres.2012.04.007.

Tartakovsky, D. M., and C. L. Winter (2008), Uncertain future of hydrogeology, J. Hydrol. Eng., 13, 37-39.

Zavala-Sanchez, V., M. Dentz, and X. Sanchez-Vila(2009), Characterization of mixing and spreading in a bounded stratified medium, Adv. Water Resour., 32, 635-648.

Zhang, Y. K., D. Zhang, and J. Lin (1996), Nonergodic solute transport in three-dimensional heterogeneous isotropic aquifers, Water Resour. Res., $32,2955-2963$ 